\title{
Analysis of Air Radiation Measurements Obtained in the EAST and X2 Shocktube Facilities
}

\author{
A. M. Brandis, ${ }^{*}$ \\ Center for Turbulence Research, Stanford University, CA, USA \\ B. A. Cruden, ${ }^{\dagger}$ D. Prabhu,,${ }^{\ddagger}$ \\ ELORET Corporation, Sunnyvale, CA, USA \\ D. Bose,,$^{\S}$ \\ NASA Ames Research Center, Mountain View, CA, USA \\ and \\ M. McGilvray, ${ }^{\top R}$. G. Morgan," \\ University of Queensland, QLD, Aus
}

\begin{abstract}
This paper presents measurements of equilibrium radiation obtained in the NASA Ames Research Center's EAST facility and the University of Queensland's X2 facility. These experiments were aimed at measuring the level of radiation encountered during conditions relevant to Orion lunar return into Earth's atmosphere. The facilities have targeted the same nominal test conditions of $10 \mathrm{~km} / \mathrm{s}$ and $26.6 \mathrm{~Pa}(0.2$ Torr $)$. In addition, variations on the nominal shock speed have also been the focus of recent testing in the EAST facility. A comprehensive comparison between the EAST data and NEQAIR is presented in this paper with preliminary X2 comparisons where appropriate. Since the two facilities have different dimensions, and the tests have different shock speeds, NEQAIR simulations are used as a point of reference for the EAST and X2 comparison. Results obtained by independently reducing the data from both facilities are compared. The present analysis endeavors to provide a better understanding of the uncertainty in the measurements, as well as provide an initial comparison between EAST and X2. Furthermore, the present analysis explores various radiative mechanisms to determine if they are due to physical processes relevant to flight, or are just facility dependent phenomena. These phenomena include effects such as the magnitude of the background continuum.
\end{abstract}

\section{Introduction}

The Vision for Space Exploration has renewed interest in understanding the radiation that is encountered during re-entry into Earth's atmosphere. In particular, the focus has been to consider conditions that are relevant to the Orion capsule, as part of the Crew Exploration Vehicle (CEV) Aerosciences Project (CAP). Due to the radiation being a significant portion of the heat flux, although only for a relatively short time in the trajectory, detailed simulations and experiments have been undertaken to quantify this radiation and

\footnotetext{
*Postdoctoral Fellow, AIAA Member, USA; aaron.m.brandis@nasa.gov

${ }^{\dagger}$ Senior Research Scientist, Senior Member AIAA

${ }^{\ddagger}$ Senior Research Scientist, Associate Fellow AIAA

$\S$ Senior Research Scientist, Associate Fellow AIAA

ฯPostdoctoral Fellow, now at Oxford University, AIAA Member

" Director for Centre of Hypersonics, AIAA Member
} 
associated uncertainties. ${ }^{1-3}$ Previous studies have identified significant differences between the experimental measurements taken in the Electric Arc Shock Tube (EAST) facility at the NASA Ames Research Center ${ }^{4}$ and theoretical models, ${ }^{2}$ such as NEQAIR ${ }^{5}$ and HARA. ${ }^{6}$ Understanding these uncertainties are therefore critical in the design of the thermal protection system (TPS). The present analysis should help provide an insight to the level of fidelity of the theoretical models, and any previous discrepancies that have already been identified, such as the level of the background continuum.

\section{Description of the Facilities}

The basic operating principle of both facilities, EAST and X2, is the same, in that the heated test gas behind the shock front simulates conditions behind the bow shock on a re-entry vehicle. This enables the experiments to be performed with flow parameters, such as velocity, static pressure, and atmospheric composition close to actual flight conditions. As the tests are performed at real flight pressures, full capsule geometry would have to be used if it is intended to test aerodynamic features of the flow. However, the objective is to study and characterize the emitted radiation, and so the aerodynamic features are not relevant for this purpose. The region of valid test gas lies between the shock front and the contact surface that separates the driver and driven gases. The test time is defined as the axial distance between these two points divided by the local shock velocity. Though short, this test time is often sufficient to capture the peak of the nonequilibrium shock radiation and the decay to equilibrium conditions. To date, there have been relatively few attempts to measure the radiative intensity of Earth entry conditions.

\section{A. EAST}

The EAST facility at NASA Ames Research Center was developed to simulate high-enthalpy, real gas phenomena encountered by hypersonic vehicles entering planetary atmospheres. It has the capability of producing superorbital shock speeds using an electric arc driver with a tube diameter of $10.16 \mathrm{~cm} .^{7,8}$ The facility was built in the late 1960s to support research in aerothermochemistry of hypervelocity flight through Earth and planetary atmospheres. The use of an electric arc discharge as the driver mechanism permits generation of shock speeds up to $46 \mathrm{~km} / \mathrm{s}$ in $\mathrm{H}_{2} / \mathrm{He}$ atmospheres. ${ }^{7,8}$ The characteristics of the EAST arc driver typically result in test times of $4-10$ microseconds. As this gas arrives at the location of the test section in the tube, a spectrometer attached to an Intensified Charge Coupled Device (ICCD) is gated and the spectral and spatial emission of the gas is analyzed. For the recent test campaign, experiments in the EAST facility have utilized a buffer gas section to obtained the higher speeds $(>10.5 \mathrm{~km} / \mathrm{s})$ presented in this paper. The purpose of the buffer gas is to slow down the arrival of the contact surface between the driver and driven gases, thus increasing the test time. Furthermore, the buffer gas arrangement has the possibility to be optimized to produce even higher speeds. The existence of contaminant species, usually carbon or hydrogen, resulting from the arc process have been identified in EAST. In the latest test campaigns of EAST, many upgrades to the system have been made in order to reduce the level of contamination. This has been very successful, however, traces of the contamination can still occasionally be identified in the spectra. EAST utilizes four spectrometers per shot, associated with four different wavelength ranges. These cameras are referred to as $\operatorname{VUV}(<\approx 215 \mathrm{~nm}), \mathrm{UV} /$ Vis $(\approx 190 \mathrm{~nm}-500 \mathrm{~nm}), \operatorname{Vis} / \mathrm{NIR}(\approx 480 \mathrm{~nm}-900 \mathrm{~nm})$ and IR $(\approx 700 \mathrm{~nm}-$ $1700 \mathrm{~nm})$.

\section{B. X2}

Additional experimental data shown in the present paper were obtained on the X2 high enthalpy shock tube facility at the University of Queensland (UQ). The two main differences between X2 and EAST are that the pressure increase in the driver gas is not caused by an electric arc, but rather by a free piston compression of the gas. In this configuration, the free piston driver is used to generate a strong shock wave that passes through the secondary driver (if required), then through the stagnant test gas in the shock tube. The secondary shock heated driver is sometimes needed in order to reach the appropriate shock velocities. The other major difference is that the test gas is not observed through a window in the side of the tunnel, but rather just after the flow has exited the tube and into the dump tank. This means that the flow-field is substantially more complex, however could offer the benefit of a reduced boundary layer effect as it has been quenched by an expansion fan process at this point. The most important dimension with regards to the experimental testing is the bore of the acceleration tube which is $85 \mathrm{~mm}$, of which approximately 70 
to $80 \mathrm{~mm}$ is the usable width of radiating gas. Contamination in the X2 facility appears to be less than EAST due to less contamination arising from the free-piston compression than the arc. However, carbon and hydrogen have also been identified in the X2 spectra. It is interesting to note that carbon contamination in the form of the cyanogen radical $(\mathrm{CN})$, has only been identified when viewed just at the exit of the tunnel, indicating that $\mathrm{CN}$ is coming off the walls and located in the boundary layer. Atomic carbon contamination has not been observed in X2 as it is outside of the wavelength range available on X2 instrumentation. X2 utilizes two spectrometers per shot, associated with two different wavelength ranges. These cameras are referred to as Blue $(\approx 300 \mathrm{~nm}-500 \mathrm{~nm})$ and Red $(\approx 500 \mathrm{~nm}-850 \mathrm{~nm})$.

\section{Test Conditions}

The nominal test conditions were chosen to be representative of Orion's re-entry into Earth's atmosphere. This corresponds to a shock speed of approximately $10 \mathrm{~km} / \mathrm{s}$ and pressures ranging from $13.3 \mathrm{~Pa}$ (0.1 Torr) to $53.2 \mathrm{~Pa}$ (0.4 Torr). Therefore, the majority of shots were aimed at producing a $10 \mathrm{~km} / \mathrm{s}$ shock. However, in attempting to achieve the nominal $10 \mathrm{~km} / \mathrm{s}$ condition, a range of approximately $9.5 \mathrm{~km} / \mathrm{s}$ to $10.5 \mathrm{~km} / \mathrm{s}$ was obtained in EAST, and approximately $9.5 \mathrm{~km} / \mathrm{s}$ to $9.7 \mathrm{~km} / \mathrm{s}$ in X2. Furthermore, in order to better ascertain the dependency of the radiation on shock speed, shots were aimed at expanding the shock speed testing conditions to encompass from $8 \mathrm{~km} / \mathrm{s}$ to $12 \mathrm{~km} / \mathrm{s}$ in EAST. This helped provide a more complete picture to analyze the relationship between shock velocity and emitted radiation. Furthermore, expanding the shock speed domain was important for the comparison with X2 as the shock speed was less than $10 \mathrm{~km} / \mathrm{s}$ (around $9.6 \mathrm{~km} / \mathrm{s}$ ) at $26.6 \mathrm{~Pa}$.

\section{Key Differences in Absolute Radiance Calibration}

The source used for the absolute intensity calibration of both the X2 and EAST results is different. For the X2 calibration, a tungsten-halogen OL200M spectral calibration lamp from Optronic Laboratories was used, whereas on the EAST facility, an integrating sphere from Spheroptics with NIST traceable absolute radiance calibration is employed. Furthermore, both calibration sources are intended as standards for different units of absolute radiometry. The UQ lamp is calibrated as an irradiance source (meaning that the solid angle captured during an experiment needs to be calculated), while EAST uses a radiance source (thus the solid angle is intrinsic to the quoted calibration). The method for calculating the zero level is also different. In EAST a "dark" count picture is taken and subtracted from both calibration and shot data. This picture allows for the level of counts on each pixel to be found when there is no light source illuminating the slit. This method assumes that there is no stray light entering the slit during the experiment. In X2, the dark counts are taken from the region in front of the shock front. The approach used in X2 has the advantage of being able to correct for any stray light that has entered the spectrometer, but has the disadvantage of assuming all sources of background to have no spatial dependence on the pixel array and may introduce errors if there are any real sources of radiation (such as reflections) ahead of the shock.

The effect of non-reciprocity, is also addressed differently in the two facilities. On EAST, different exposure times for the calibration and the experiment are used and the experimental exposures can be several orders of magnitude smaller than those for calibration. This is done so the level of the counts between experiment and calibration are approximately the same. A correction factor is then employed to correct for the effect of non-reciprocity. In X2 the same exposure time is used for the experimental and calibration image and thus avoids any non-reciprocity effects. However, because the calibration picture has a small exposure time, the number of counts can be very low, especially in the Blue region of the spectrum. This also requires the $\mathrm{X} 2$ calibrations to bin the pixel rows from the CCD and thus prevents the calibration from being corrected due to non-uniformity in the CCD. Therefore, UQ relies on linearity of the pixel counts versus intensity and lack of spatial dependence on the CCD and accepts an increased level of uncertainty on the Blue calibration, while EAST relies on the linearity of pixel counts versus intensity and the reciprocity function being constant and well known.

Another difference regarding the calibration is the way the optical arrangement is configured between the two different procedures. On the EAST facility, the integrating sphere is placed at a location representative of the center-line of the tunnel, and the emitted light is captured by exactly the same optical arrangement as used in the experiment. However, the X2 calibration procedure is to place the lamp directly in front of the spectrometer with no intervening optics. The losses of the intervening optics then have to be measured 
and taken into account, e.g. optical magnification, losses due to mirrors and windows. The calibration is conducted in this way due to the use of a different calibration source.

\section{Experimental Results}

\section{A. Equilibrium condition assumption}

A critical aspect of these shock tunnel experiments is whether or not the shocked flow reaches thermodynamic equilibrium conditions. Due to the short time-scales and complex flow found in shock tunnels, thermodynamic equilibrium is neither guaranteed nor easily quantifiable. An analysis was undertaken to attempt to assess the level of equilibrium that was achieved in the EAST and X2 facilities. The analysis examined the evolution of the background subtracted ratios of various integrated line intensities at distances behind the shock where equilibrium is thought to exist. The background subtraction comprises of removing all the radiation emitted by the background continuum, leaving the radiation emitted only by atoms and molecules, as represented in Fig. 1. The integration process is represented by equation 1, where $I_{s f}$ is the integrated value of the spectral feature, $\lambda_{c}$ is the central wavelength of the integration domain and $I_{\lambda \text { min }}$ is minimum intensity in the specified wavelength domain.

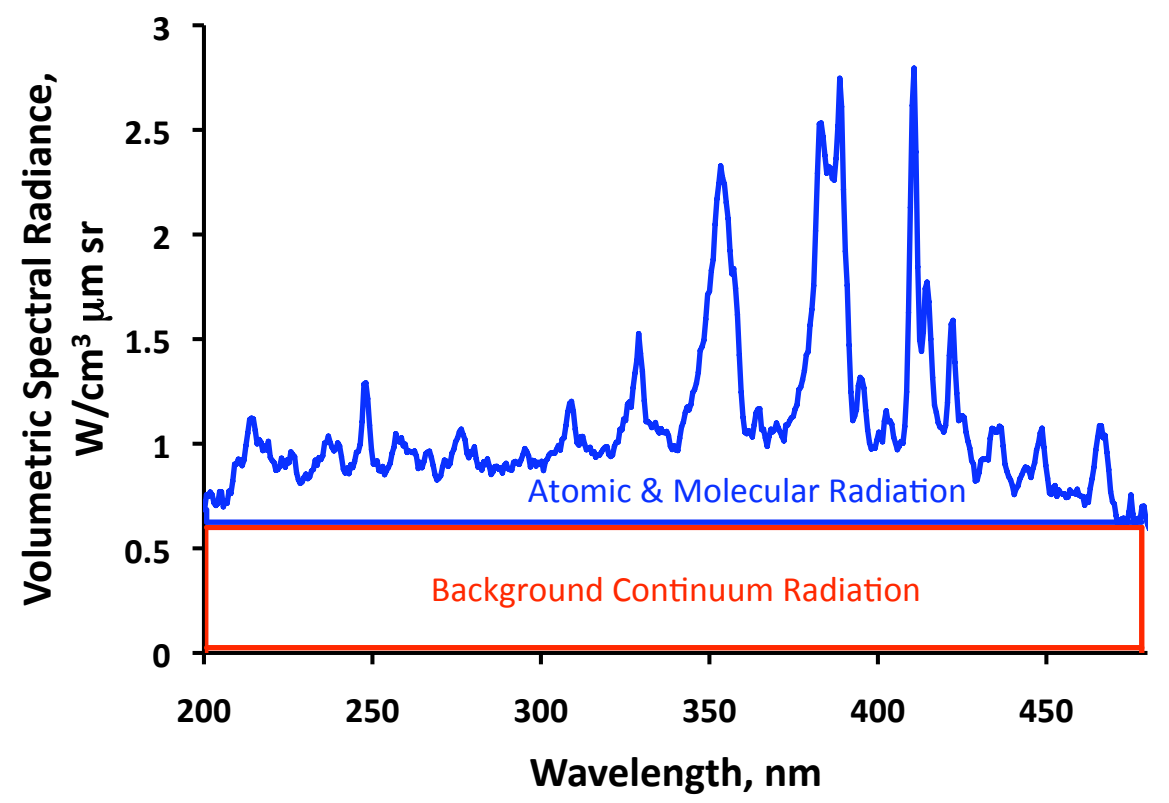

Figure 1. Representation of the radiation emitted by the background and by atomic and molecular radiation.

$$
I_{s f}=\int_{\lambda_{c}-k \times F W H M}^{\lambda_{c}+k \times F W H M}\left(I_{\lambda}-I_{\lambda \min }\right) \delta \lambda
$$

Table 1. Wavelength Range/FWHM (k) for each spectral range integrated and corresponding upper and lower state energies for each transition.

\begin{tabular}{ccccc}
\hline \hline Facility & $\begin{array}{c}\text { Spectral Feature } \\
\text { Location, nm }\end{array}$ & $\begin{array}{c}\text { Wavelength Range } \\
\text { /FWHM (k) }\end{array}$ & $\begin{array}{c}\text { Energy: } \\
\text { Lower State, } \mathbf{c m}^{-1}\end{array}$ & $\begin{array}{c}\text { Energy: } \\
\text { Upper State, } \mathbf{~ c m}^{-1}\end{array}$ \\
\hline \multirow{2}{*}{ EAST } & 777 & 10 & 73768.200 & 86629.089 \\
& 820 & 15 & 95159.649 & 107349.651 \\
& 845 & 7 & 76794.978 & 88630.977 \\
X2 & 745 & 22 & 92329.109 & 106018.848 \\
& 777 & 22 & 73768.200 & 86629.089 \\
\hline \hline
\end{tabular}


The domain over which each spectral range is integrated is specified by the ratio of the Wavelength Range/FWHM (Full Width Half Max) and is defined as, $k$. The integration domain was extended as large as possible to include all the emitted radiation, but can be limited by other nearby spectral features. The values for $k$ used in this analysis are shown in Table 1. Fig. 2(a) shows how the ratio of both the integrated line intensities of $820 \mathrm{~nm}$ (predominantly nitrogen, with some oxygen) and $845 \mathrm{~nm}$ (oxygen) with $777 \mathrm{~nm}$ (oxygen) behind the shock. The values for $k$ are different for EAST and X2 due to the different gratings/spectral resolution used for the respective experiments. The experimental values can then be compared with theoretical values. Ratios have been used as an equilibrium indicator, as opposed to an absolute measurement, to remove quantities from the analysis that maybe changing with time, such as flow density and pressure, or effect of the deceleration of the shock, or uncertainties due to calibration. As Fig. 2(a) shows, the agreement between the EAST data and theory is very good with the ratio being very constant with respect to distance behind the shock. This indicates that at least partial local equilibrium has been reached, even though the flow is not in full thermo-chemical equilibrium. A similar analysis was conducted with the X2 data using the integrated line intensities of $777 \mathrm{~nm}$ (oxygen) and $745 \mathrm{~nm}$ (nitrogen), and this is shown in Fig. 2(b). The reason for the use of different wavelength ranges was due to the different grating settings used compared to EAST. The agreement is initially very good on X2 until around $2 \mathrm{~cm}$ behind the shock. After this distance, there is a significant deviation from the theoretical value. This could suggest that at this location the expansion fan is interrupting the local thermodynamic equilibrium.

\section{Analysis of EAST Results}

The objective of this section is to present the results of a thorough analysis of the experimental data obtained in the EAST facility. Results of NEQAIR simulations for a post-shock equilibrium state serve as a reference. All of the spectral features observed in the EAST facility, except for contamination species, have been individually analyzed. The benefit of such an analysis, as opposed to comparing the spectrally integrated value, is that individual discrepancies can be identified. Furthermore, spectral features that are known to be due to contamination, can be identified and not included in the analysis. It should also be pointed out that the present analysis attempts to remove the background continuum from all calculations, and focus purely on the radiance emitted by spectral features. The observed background continuum has yet to be satisfactorily explained, and it is not completely known what mechanism is the source of this radiation and thus can not be included. Due to the large number of shots performed in the EAST facility, there is an excellent basis for a statistical analysis of the data. In this way, the effect of shot-to-shot variation can be seen, and an idea of the level of scatter can be assessed. Furthermore, individual shots can be identified as "outliers" and radiance trends can be seen with respect to shock velocity.

In order to develop an accurate assessment of how well NEQAIR simulates measured data, it is not necessary to base this assessment on every single shot in each test campaign. Due to the variability in the driver, some shots do not reach an adequate plateau region that is designated to be in equilibrium. In some cases, there is no, or very little, test time. Therefore, to better analyze the EAST data, a criterion for classifying each spectral data set was established. Each spectral data set is assigned a value indicating the "quality" of equilibrium, or plateau, achieved. An example of categories 5 to 2 is shown in Fig. 3. The classification of each shot, based on expert judgement, however, is as follows:

- "5": The spectrally integrated radiance shows a long and constant plateau region,

- "4": The spectrally integrated radiance shows a clear, but shorter constant plateau region,

- "3": The spectrally integrated radiance shows a reasonably good plateau region, however shows larger noise, a slight slope, or is very short.

- "2": The spectrally integrated radiance shows no clear plateau region, or significantly slope or noise.

- "1": Hard to even determine where the plateau region would be, potentially indicating no test time for the shot. Or the spectrally integrated radiance is rising steeply the whole time.

\section{A. Cross Section Procedures}

The volumetric radiance is obtained for each camera and shot, and the "equilibrium" plateau region is taken through inspection of each data set. Volumetric radiance is defined as the amount of power emitted per unit 

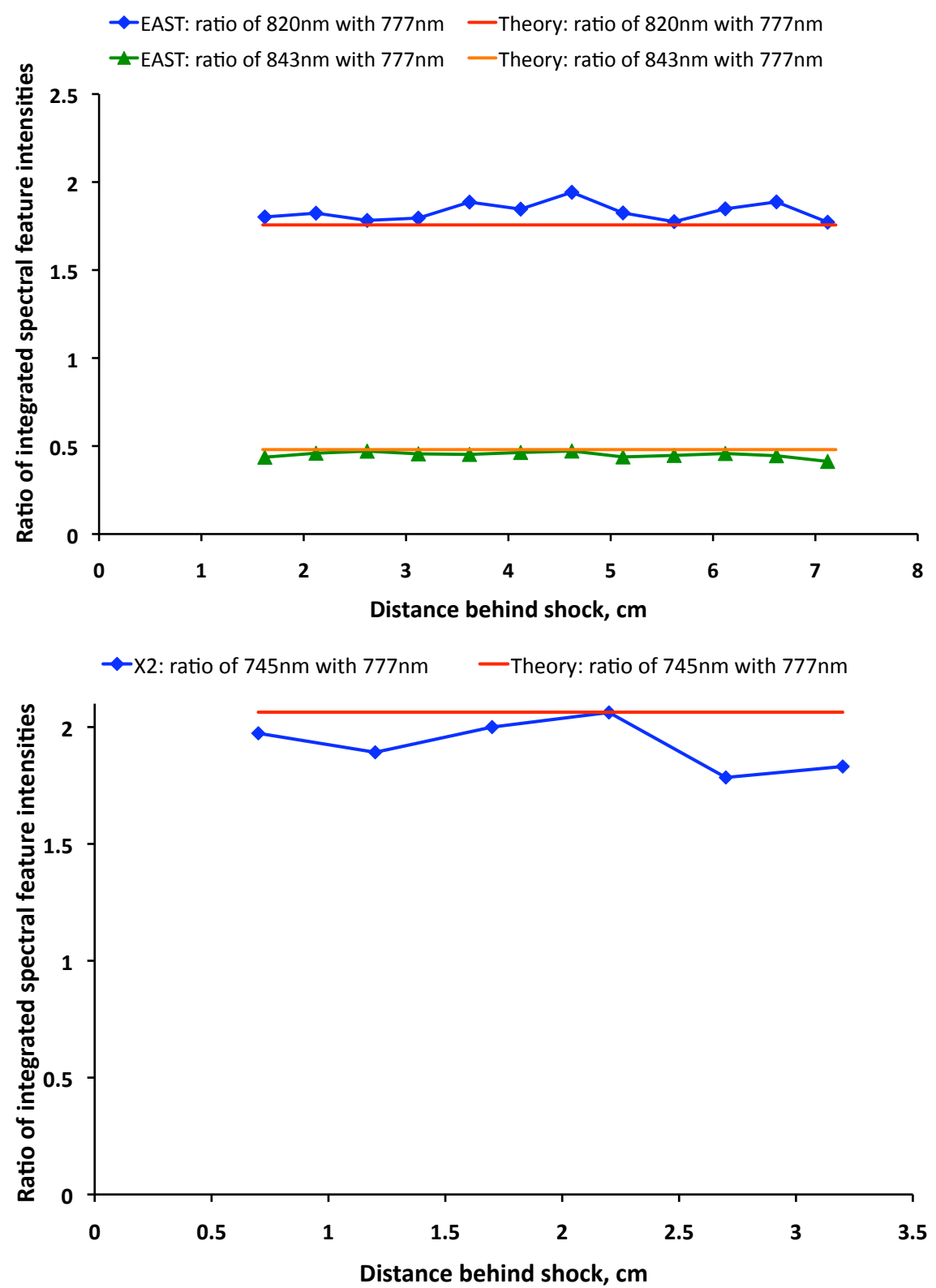

Figure 2. Comparison of the ratio of the integrated intensities at different axial positions behind the shock with theory for the (a) $820 \mathrm{~nm}, 845 \mathrm{~nm}$ and $777 \mathrm{~nm}$ on EAST, and, (b) $777 \mathrm{~nm}$ and $745 \mathrm{~nm}$ on X2. 


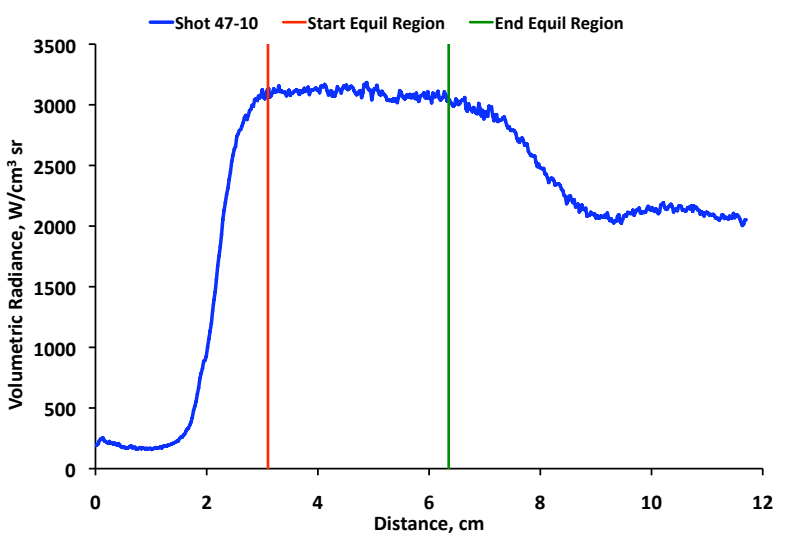

(a)

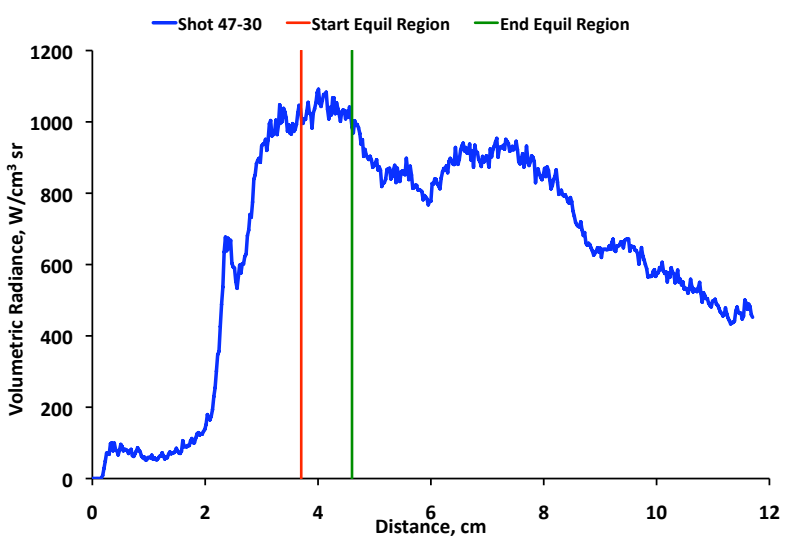

(c)

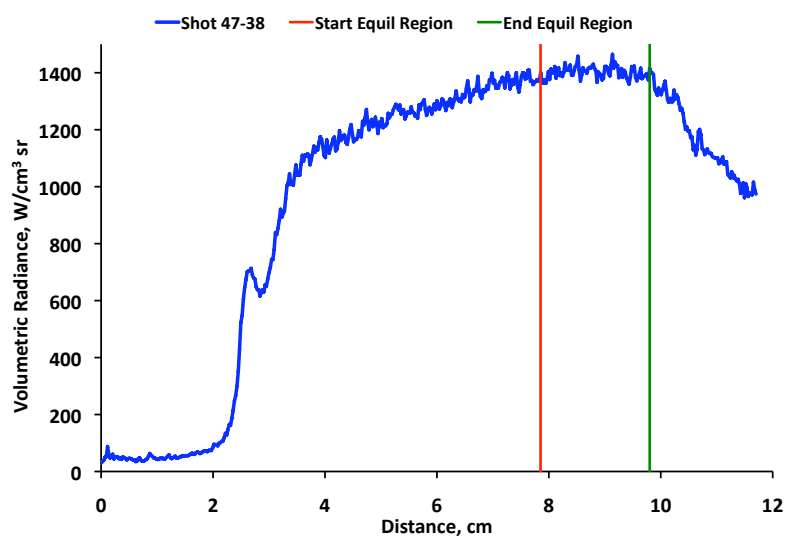

(b)

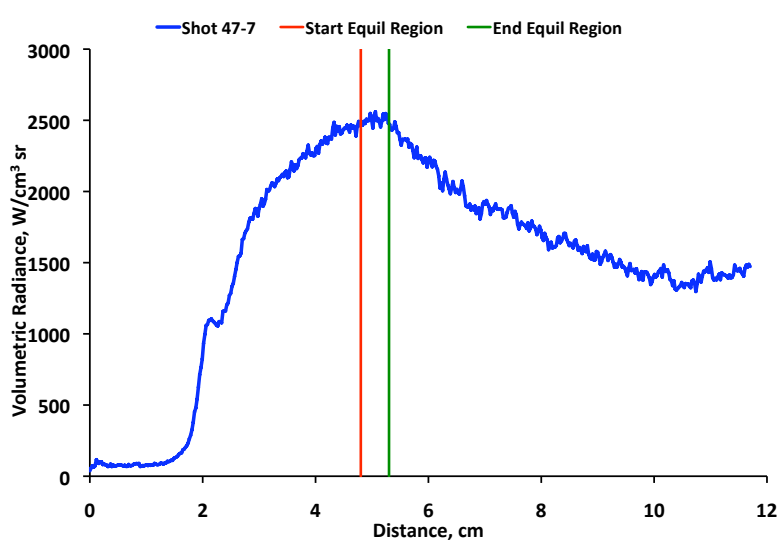

(d)

Figure 3. Examples of the equilibrium plateau region criteria for level (a) 5, (b) 4, (c) 3, and (d) 2. 
volume per solid angle $\left(\mathrm{W} / \mathrm{cm}^{3} \cdot \mathrm{sr}\right)$. To obtain the volumetric radiance, the calibrated spectral radiance data $\left(\mathrm{W} / \mathrm{cm}^{2} . \mu \mathrm{m} . \mathrm{sr}\right)$ from the experiment is normalized by the core flow width and integrated over wavelength. As was outlined above, this equilibrium plateau is more pronounced for some shots compared to others. Various spectral features are then identified in the cross section. The cross section being the profile of the averaged volumetric spectral radiance $\left(\mathrm{W} / \mathrm{cm}^{3} . \mu \mathrm{m} . \mathrm{sr}\right)$ in the equilibrium region. For each spectral feature, the minimum value in the wavelength region of interest is subtracted in an attempt to remove the background continuum. The background subtracted spectral feature intensity is then integrated over wavelength. The advantage of comparing the integrated spectral feature, as opposed to comparing spectra, is that it removes any differences relating to spectral resolution. With these data available, the integrated radiance for various spectral features can be plotted for each shot performed on EAST and compared with NEQAIR.

When integrating spectral features, one must be aware of the error associated with the integration. Firstly, to attempt to minimize this error, and secondly to understand the magnitude of this error. The error can be originate from several different sources, such as:

- The wavelength range chosen for integration of spectral radiance as this range is not always easily defined. Due to different grating settings, the discretization of the wavelength range with pixels, and small misalignments of the grating, the wavelength required for integration of a designated spectral feature can change slightly from shot to shot. Furthermore, the range needs to be selected so intensity from a nearby spectral feature does not impinge on the region of interest. It is for reasons such as this, that impedes the automation of the process, and is therefore labour intensive, relying on expert judgement.

- To get an accurate integration of the spectral features, the background continuum radiation must be subtracted from the experimental results. Assigning the level of this background is not always straightforward because the mechanisms responsible for the background continuum are not well understood. At present, the integrated spectral regions are chosen such that the minimum value in that region is representative of the continuum. This method is not ideal for a couple of reasons. Firstly, in doing this there is a possibility that part of the line intensity has also been in subtracted, and secondly, the background subtraction is only as precise as the level of noise. The best case would be to subtract the true background continuum, but unfortunately at present, the structure of this continuum is presently not well understood, so is not a plausible option. This is why the local minimum is used as an estimate of the background continuum. The goal is to select wavelength ranges that would minimize the effect of losing spectral feature radiation to the background subtraction. Thus, a spectral radiance equal to the worst case noise value, as well as any slope in the continuum, gets integrated into the volumetric radiance. However, these two factors will offset each other, making it difficult to be any more precise than this. This assumption is quite accurate in certain locations in the VUV and Vis/NIR regions, but less so in the UV/Vis.

\section{B. NEQAIR Code}

NEQAIR is a line-by-line radiation code which stands for Nonequilibrium Air Radiation. NEQAIR computes spontaneous emission, absorption and stimulated emission due to transitions between various energy states of chemical species along a line-of-sight. Individual electronic transitions are considered for atoms and molecules, with the molecular band systems being resolved for each rotational line. For more detail about NEQAIR, please refer to published literature..$^{5,9}$ The process for conducting the NEQAIR simulations is to perform the calculation at a very high spectral resolution followed by a convolution (scan) with a slit function. The slit function is a Gaussian function with the FWHM set to the line width of the measured line shapes. The line and band systems used in the radiative heating computation are shown in Table 2. It should be noted that the simulations for modelling the radiation measured on the EAST facility are an idealized approximation of the actual tube flow phenomena. Firstly, the NEQAIR simulation is based on an assumption that the flow is in equilibrium, with the post-shock conditions being calculated by CEA (Chemical Equilibrium with Applications). ${ }^{10}$ Due to the complex nature of the shock tube environment, there is no guarantee that the EAST flow actually reaches thermodynamic equilibrium. Furthermore, the idea of "measuring" the level of equilibrium is very difficult, if not impossible, in this situation. Therefore, this approximation could lead to discrepancies between the simulation and measured results. The "equilibrium" zone is stated to be the region where the integrated radiance reaches a plateau. The equilibrium flow 
environment is also calculated based on the speed of the shock front at the test section. It therefore does not take into account that the shock is decelerating. This deceleration of the gas is important as the gas in the "equilibrium" region would of been shocked at different velocities. However, as eluded to earlier, it is possible that there are sections of gas that are in a local equilibrium, but the entire test gas overall is not in equilibrium. In the test section, the shock can be decelerating at approximately $500 \mathrm{~m} / \mathrm{s}^{2}$ (as an example from $10.1 \mathrm{~km} / \mathrm{s}$ to $9.92 \mathrm{~km} / \mathrm{s}$ ), which can correspond to a decrease in radiance of approximately $25-30 \%$ in the Vis/NIR or $35 \%$ in VUV region of the spectrometer. Further discrepancies could arise due to the calculation being a one-dimensional solution, and as such does not include the effect of the boundary layer on the wall of the shock tube. Therefore absorption in the boundary layer is not accounted for in the analysis. A time-accurate 2-D/axisymmetric simulation of the facility would be required to address these issues.

Table 2. Line and band systems for radiative heating computations using NEQAIR

\begin{tabular}{cc}
\hline \hline Species & Systems \\
\hline $\mathrm{N}$ & lines/multiplets between $120 \mathrm{~nm}$ and $1.5 \mu \mathrm{m}$ \\
$\mathrm{O}$ & lines/multiplets between $120 \mathrm{~nm}$ and $1.5 \mu \mathrm{m}$ \\
$\mathrm{N}^{+}, \mathrm{O}^{+}, \mathrm{e}^{-}$ & bound-free and free-free continua \\
$\mathrm{N}_{2}$ & $1^{\text {st }}$-positive $\left(B^{3} \Pi_{g} \rightarrow A^{3} \Sigma_{u}^{+}\right), 2^{\text {nd }}$-positive $\left(C^{3} \Pi_{u} \rightarrow B^{3} \Pi_{g}\right)$ \\
$\mathrm{O}_{2}$ & Schumann-Runge $\left(B{ }^{3} \Sigma_{u}^{-} \rightarrow X^{3} \Sigma_{g}^{-}\right)$ \\
$\mathrm{NO}$ & $\beta\left(B^{2} \Pi \rightarrow X^{2} \Pi\right), \gamma\left(A^{2} \Sigma^{-} \rightarrow X^{2} \Pi\right), \delta\left(C^{2} \Pi \rightarrow X^{2} \Pi\right), \epsilon\left(D^{2} \Sigma^{+} \rightarrow X^{2} \Pi\right)$ \\
$\mathrm{N}_{2}^{+}$ & $1^{\text {st }}$-negative $\left(B^{2} \Sigma_{u}^{+} \rightarrow X^{2} \Sigma_{g}^{+}\right)$ \\
\hline \hline
\end{tabular}

\section{Comparison of EAST and NEQAIR}

The comparison of EAST and NEQAIR is not necessarily a trivial task, especially since one must ensure that one is comparing the same spectral phenomena in the experiment and the simulation. For example, one should not compare the integrated value of a spectral feature from the experiment that includes a large percentage of the background continuum, with a simulated integrated value that just accounts for the spectral feature intensity. If this were the case one could either get a false good agreement, or false bad agreement. Alternatively, the experimental data could contain contamination that is not included in the simulation. Care must be taken to make sure the same features are compared with integrated intensities. Furthermore, conducting this comparison alongside an appropriately convolved spectral feature analysis is also beneficial. Due to a slightly different optical set up and issues associated with each spectral range, the comparison is broken up into three of the four different cameras used during each EAST experiment. The IR camera has not been analyzed in the present study due to a recent discovery that there is an issue related to the camera saturating at counts much lower than expected. The following sections present an analysis and comparison of NEQAIR and EAST for the VUV, UV/Vis and Vis/NIR spectral regions. The goal being to assign upper and lower bounds of uncertainty regarding the agreement of the NEQAIR simulations to the EAST experimental results.

\section{1. $\operatorname{VUV}$ Spectra $(<\approx 215 \mathrm{~nm})$}

The VUV spectral region is a very difficult region to obtain data due to the absorption of the emitted radiation in this wavelength range by oxygen. The collection optics and spectrometer need to be located in a vacuum environment, and special windows are required to allow the transmission of data for wavelengths shorter than $\sim 190 \mathrm{~nm}$. The EAST experiments are currently the only source of data available for VUV spectra obtained in a shock tube for re-entry relevant conditions. The main spectral features of interest for this comparison in the VUV are the $120 \mathrm{~nm}$ and $174 \mathrm{~nm}$ nitrogen lines. A comparison of the NEQAIR and EAST data for these lines is presented in Fig. 4. The agreement is reasonably good, within upper and lower error bounds of $0 \%$ and $-40 \%$ for the $149 \mathrm{~nm}$ line, and $-10 \%$ and $-30 \%$ for the $174 \mathrm{~nm}$ line. However, what is interesting to note about this data set is that the majority of the EAST data are below the NEQAIR prediction, except for a couple of low rated and slow shots. This could indicate either that there is a systematic error in the calibration of the VUV camera, or that there is some significant physical process 
missing from the modeling of this line. For example, there are uncertainties related to the Einstein "A" coefficients (uncertainties of $\leq 7 \%$ and $\leq 10 \%$ for the $149 \mathrm{~nm}$ and $174 \mathrm{~nm}$ respectively ${ }^{11}$ ), or shock tube flow effects such as, absorption in the boundary layer or the deceleration of the shock. Further analysis of this line utilizing 2-D/axi-symmetric simulations could provide some insight into these issues. A spectral comparison of one shot from EAST and NEQAIR is shown in Fig. 5. This figure shows excellent agreement between the two lines, and also highlights that purely comparing the peaks between experiments and simulations is not always completely valid. As can be seen in the figure, NEQAIR has a greater peak height than measured on EAST. However, this is compensated by the increased level of energy in the wings of the spectral features measured on EAST. Without doing this, the integrated value of the spectral feature is the best value for comparison.

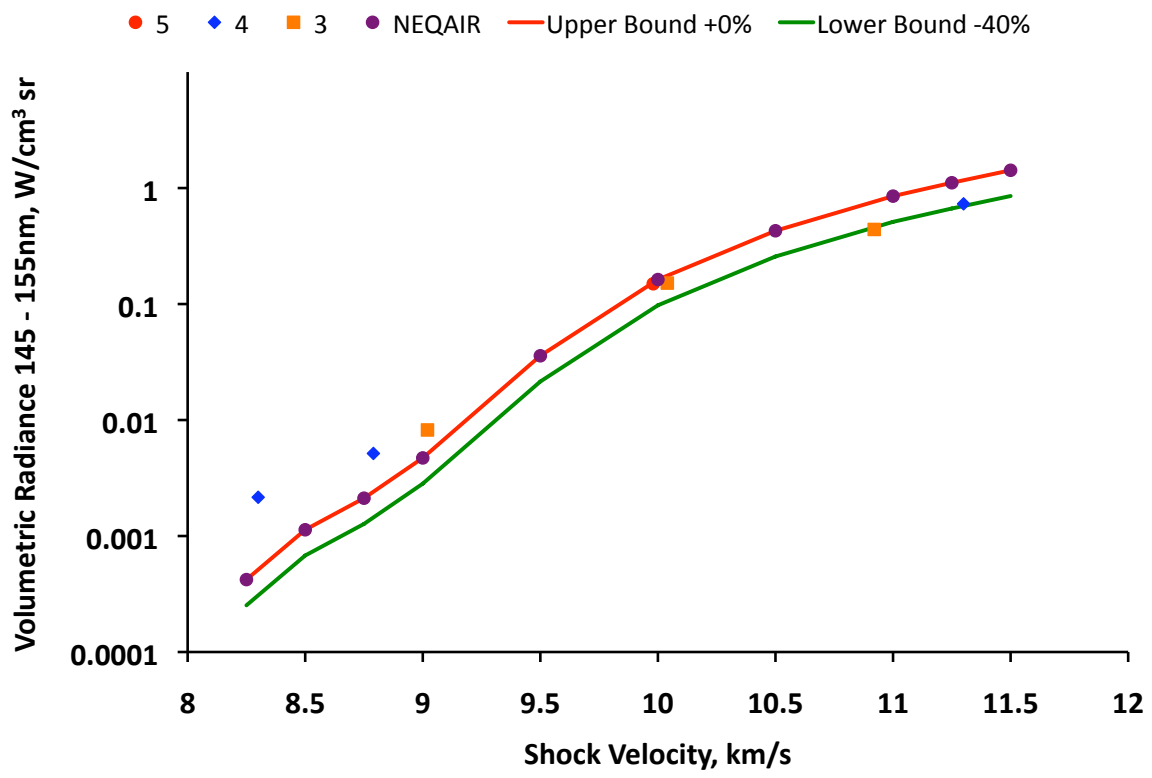

(a)

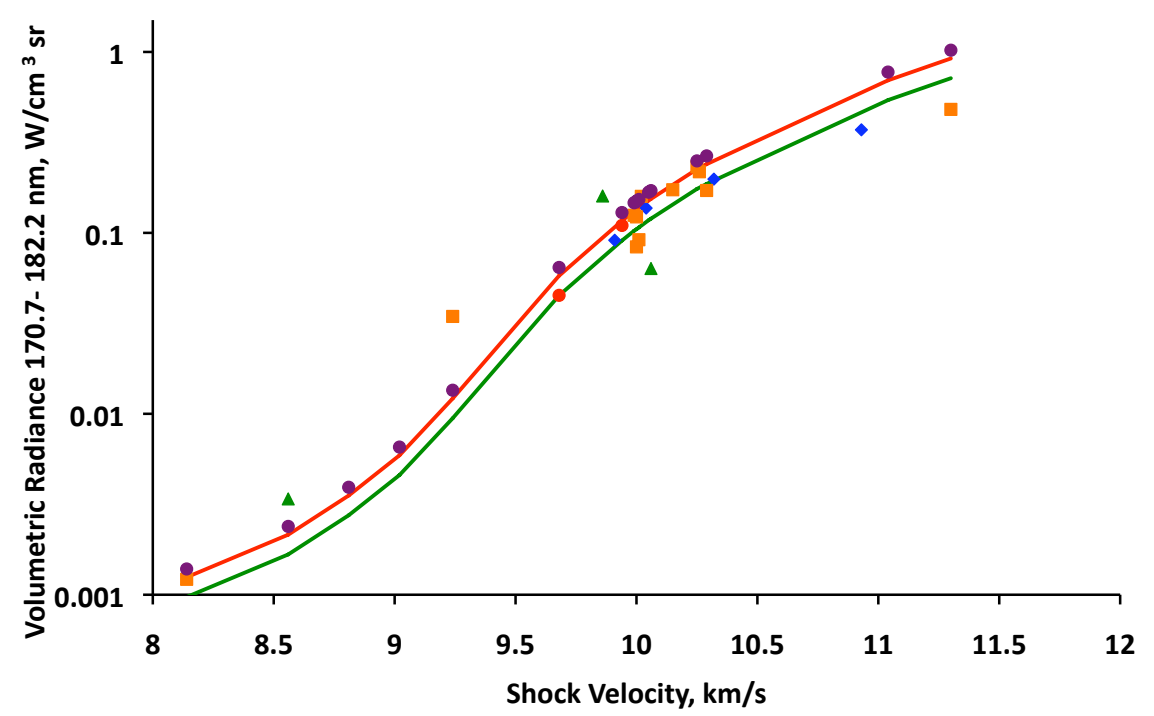

(b)

Figure 4. VUV comparisons of NEQAIR simulations and Test Campaign 47 and 50 EAST Experiments for the (a) $149 \mathrm{~nm}$ nitrogen line (log-scale) and (b) $174 \mathrm{~nm}$ nitrogen line (log-scale). 


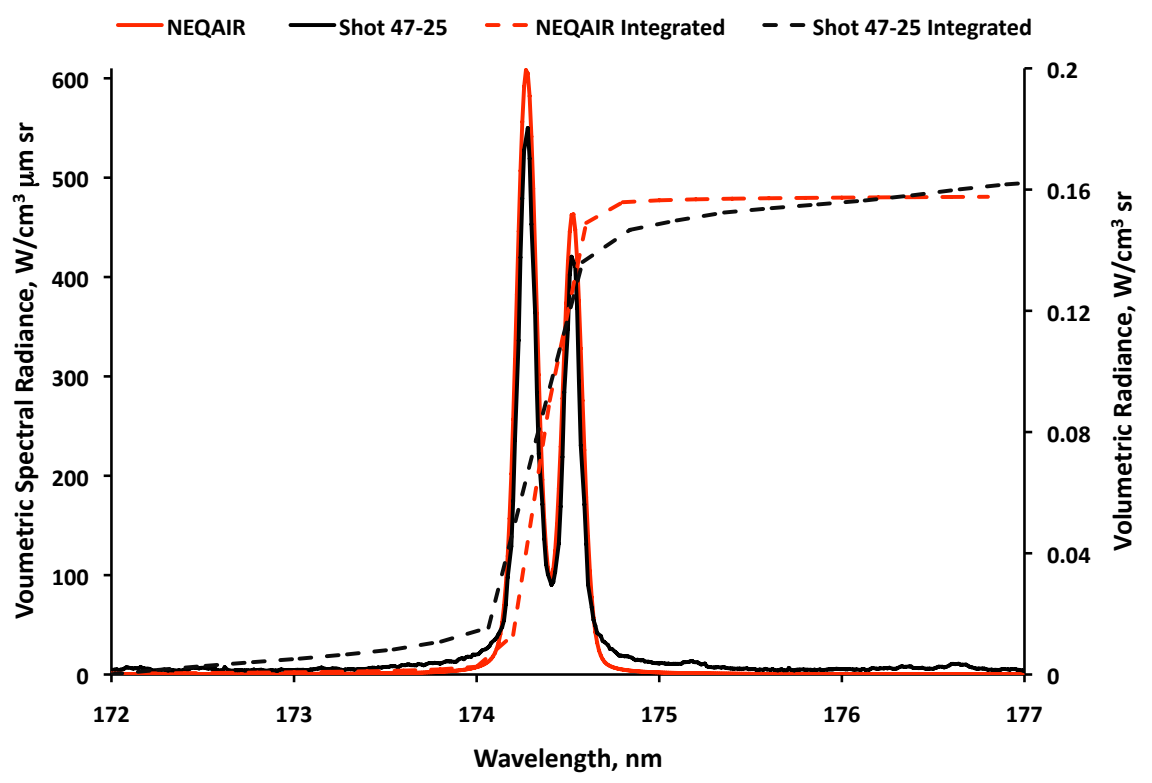

Figure 5. Comparison of NEQAIR and EAST for the $174 \mathrm{~nm}$ nitrogen line with the background continuum subtracted at $10.25 \mathrm{~km} / \mathrm{s}$.

\section{2. $U V /$ Vis Spectra $(\approx 190 \mathrm{~nm}-500 \mathrm{~nm})$}

The UV/Vis region of the spectrometer is one of the more challenging regions to simulate. This is due to the presence of molecular radiation as well as atomic radiation. The modeling of molecular radiation is more difficult due to the existence of the vibrational manifolds and branches of rotational lines. Thus the molecular radiation is a much broader phenomena in terms of wavelength. Therefore, the molecular radiation can be superimposed on the atomic radiation. Furthermore, for many of the conditions observed on EAST, the dissociation limit for some of these processes can be reached. This means that for some of the slower shots, there could be a molecular band radiating, which may not be present in a faster shot due to the temperature being much higher, and thus the molecule may have dissociated into its atomic substituents. For example, for the simulation of shot $47-43$ shown in Fig. 7, it appears that NEQAIR is predicting emitted radiance from an $\mathrm{N}_{2}$ molecular system between $425 \mathrm{~nm}$ and $430 \mathrm{~nm}$. However, this spectral feature is not observed in the EAST experiment. There are two possible causes for this discrepancy. One being that the flow has not reached the equilibrium flow-field as calculated by $\mathrm{CEA},{ }^{10}$ and so is in some level of non-equilibrium. The other possible cause being that NEQAIR's spectroscopic constants are incorrect for this feature.

The analysis of the UV/Vis region of the spectrum is also approached in a different manner to the VUV region due to the superposition of both atomic and molecular emission lines. This means that unlike the VUV region where single spectral features can be analyzed, the UV/Vis spectral region needs to be analyzed as a whole as it is very difficult to separate the various emitting features. Furthermore, due to the superposition of spectral features and broad band emissions, the subtraction of the background continuum is more difficult in this region. The comparison of the simulated and experimental radiance integrated over the region of $310 \mathrm{~nm}$ to $470 \mathrm{~nm}$ is shown in Fig. 6. The results show that NEQAIR and EAST quantitatively agree quite well from 9.7 to $11.5 \mathrm{~km} / \mathrm{s}$. Furthermore, the trend of emitted radiance with velocity agrees very well. However, when the lower speeds are analyzed the agreement is not as good, with NEQAIR under predicting the EAST results. This a common result across all spectral features, with NEQAIR under-predicting the radiation at slower speeds (approximately $8-9.5 \mathrm{~km} / \mathrm{s}$ ).

\section{Vis/NIR Spectra $(\approx 480 \mathrm{~nm}-900 \mathrm{~nm})$}

The Vis/NIR region of the spectrum is typically easier to simulate due to the radiation being emitted from atomic species only and the effect of the background continuum subtraction being less significant. This is shown in Fig. 9 where excellent agreement can be seen between NEQAIR and EAST spectrum. A comparison of NEQAIR and EAST for the integrated $777 \mathrm{~nm}$ oxygen line is shown in Fig. 8. As can be seen, 


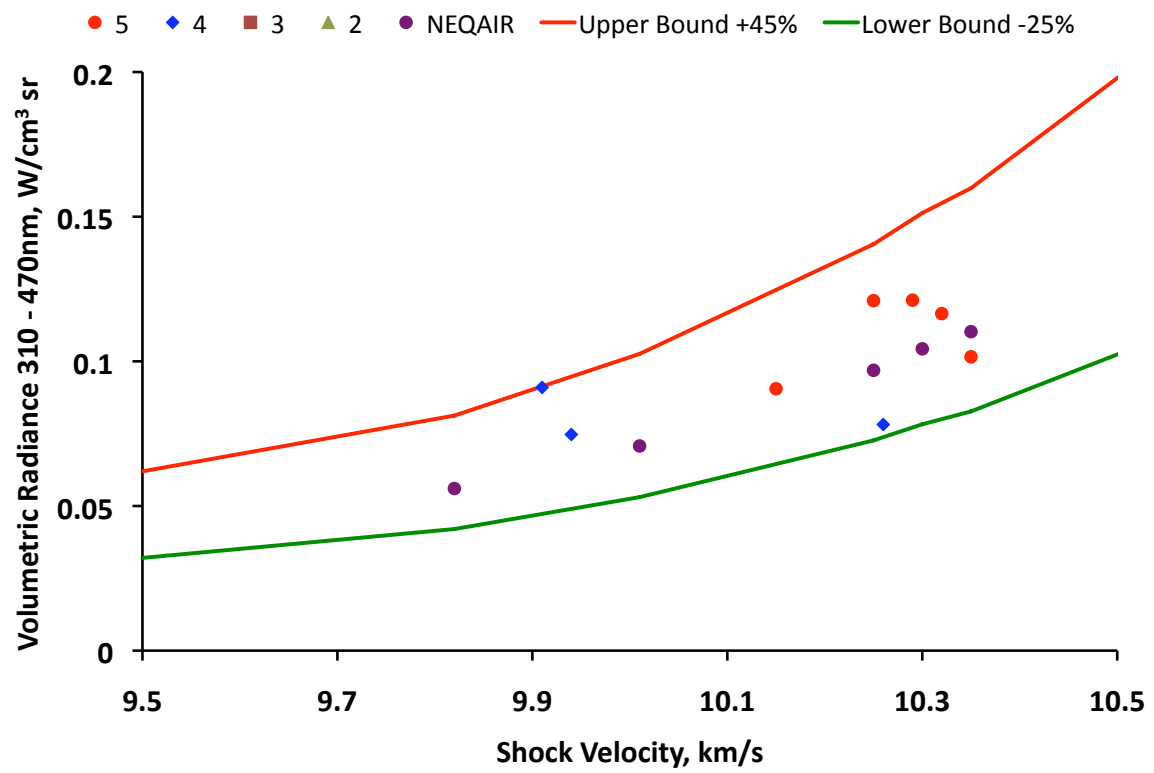

(a)

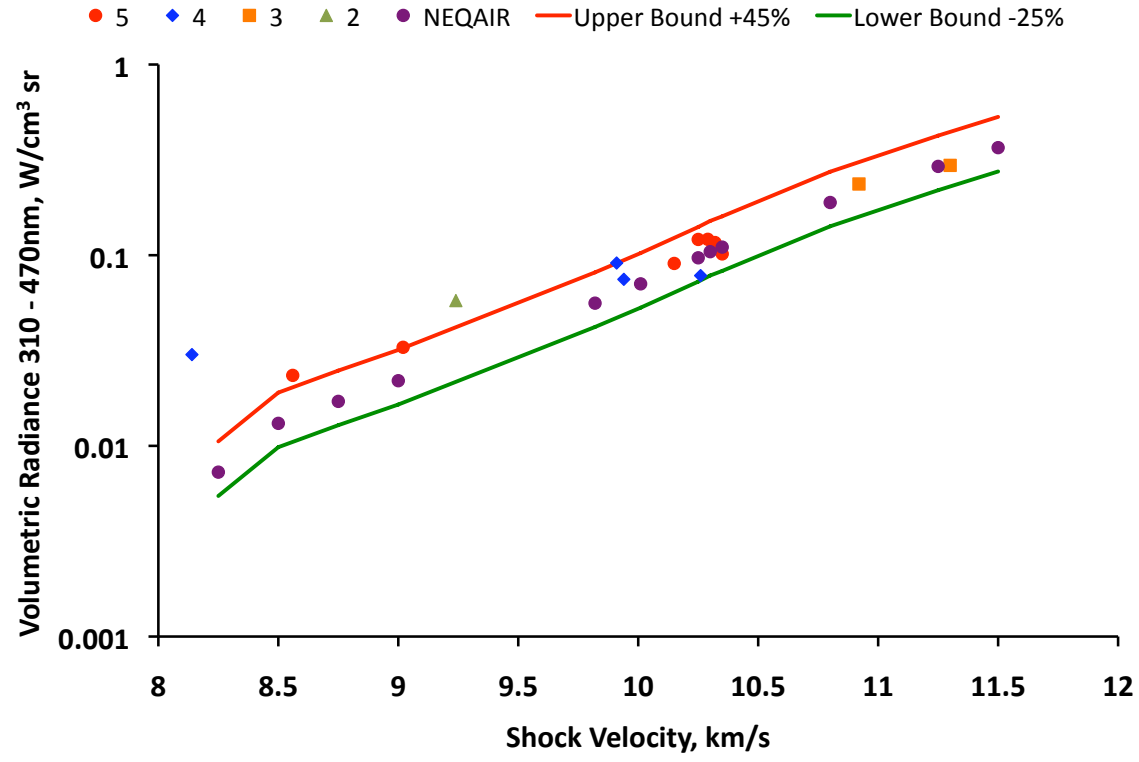

(b)

Figure 6. UV/Vis comparisons of NEQAIR simulations and Test Campaign 47 and 50 EAST Experiments for the spectral range of $310 \mathrm{~nm}$ to $470 \mathrm{~nm}$ on a (a) linear scale, and, (b) log-scale. 


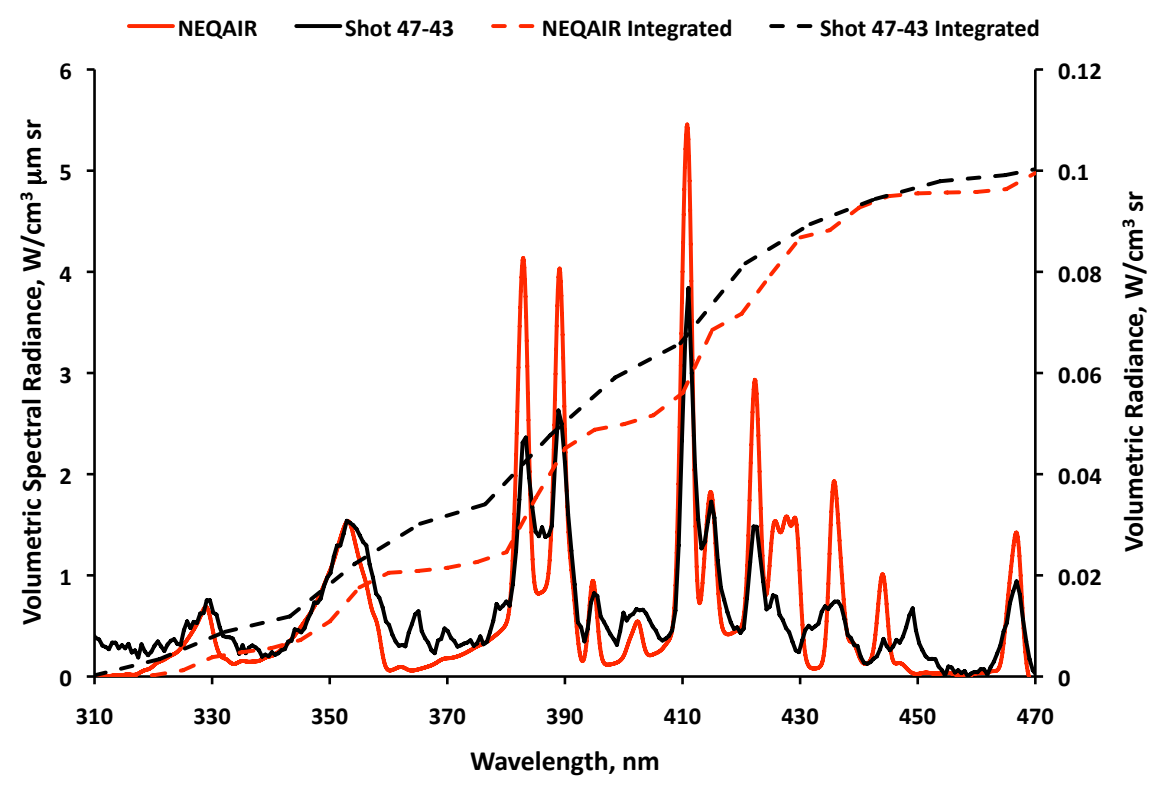

Figure 7. Comparison of NEQAIR and EAST for the spectral range of $310 \mathrm{~nm}$ to $470 \mathrm{~nm}$ with the background continuum subtracted.

the agreement is very good with upper and lower error bounds of $+5 \%$ and $-35 \%$. Furthermore, a similar trend can be identified in the Vis/NIR spectral region as in the VUV, that the NEQAIR solution almost presents an upper bound to the EAST data.

One issue that has been identified on the Vis/NIR spectrometer is an effect of non-reciprocity. ${ }^{4}$ That is, when the gate width is doubled, the spectrometer does not measure double the amount of photon counts. This is important because the calibration light source is orders of magnitude less bright than the light emitted from the shock and test gas. To compensate for this in the calibration, the gate width of the spectrometer has to be greatly increased to achieve a similar order of counts. These different exposure times then require a correction factor which differs from the ratio of the exposure times. The reason for this non-reciprocity has been assigned to the delay associated with the opening of the gate. The opening and closing of the gate has been experimentally measured by using a nano-pulsing lamp and systematically increasing the gate delay. Two examples of this experiment for gate widths of $100 \mathrm{~ns}$ and $500 \mathrm{~ns}$ are presented in Fig. 10. The level of noise is due to the variability in the lamp pulse. As can be seen from the figures, the gate opening takes place over a large time scale (approximately $1400 \mathrm{~ns}$ for the $500 \mathrm{~ns}$ gate). The process takes so long, that at the $100 \mathrm{~ns}$ gate width (which is the setting used for the majority of the experiments), it appears that the gate is never fully open.

\section{Overall NEQAIR Maximum and Minimum Uncertainty Bounds}

After the data is collected and analyzed from each camera, the goal then becomes to determine an overall upper and lower uncertainty bound for the NEQAIR prediction of EAST data. The methodology for this analysis is to assign upper and lower bounds for each spectral region analyzed. The level of the bounds have been assigned to encompass the equilibrium level rated "4" and "5" data, specifically aimed at the shots between $9.5-10.5 \mathrm{~km} / \mathrm{s}$. This shock speed range was chosen because it is the range with the most shot data as well as being the most flight relevant. The extent to which the experimental data is over or under predicted by the NEQAIR simulations is then used to determine the overall upper and lower bounds of NEQAIR'S ability to predict the EAST radiance. Once the bounds for each experimental spectral region have been determined, the influence of these uncertainties on the overall uncertainty bounds can be evaluated. This is achieved by weighting each spectral range uncertainty by the amount of radiance emitted in each specific wavelength region. The weighting is evaluated by using the NEQAIR solution at $10 \mathrm{~km} / \mathrm{s}$ as the baseline. For each spectral range, the percentage of the emitted radiation in the that range compared to the overall radiation emitted is the weighting factor. The weighting factors can then be used as a multiplier for each 


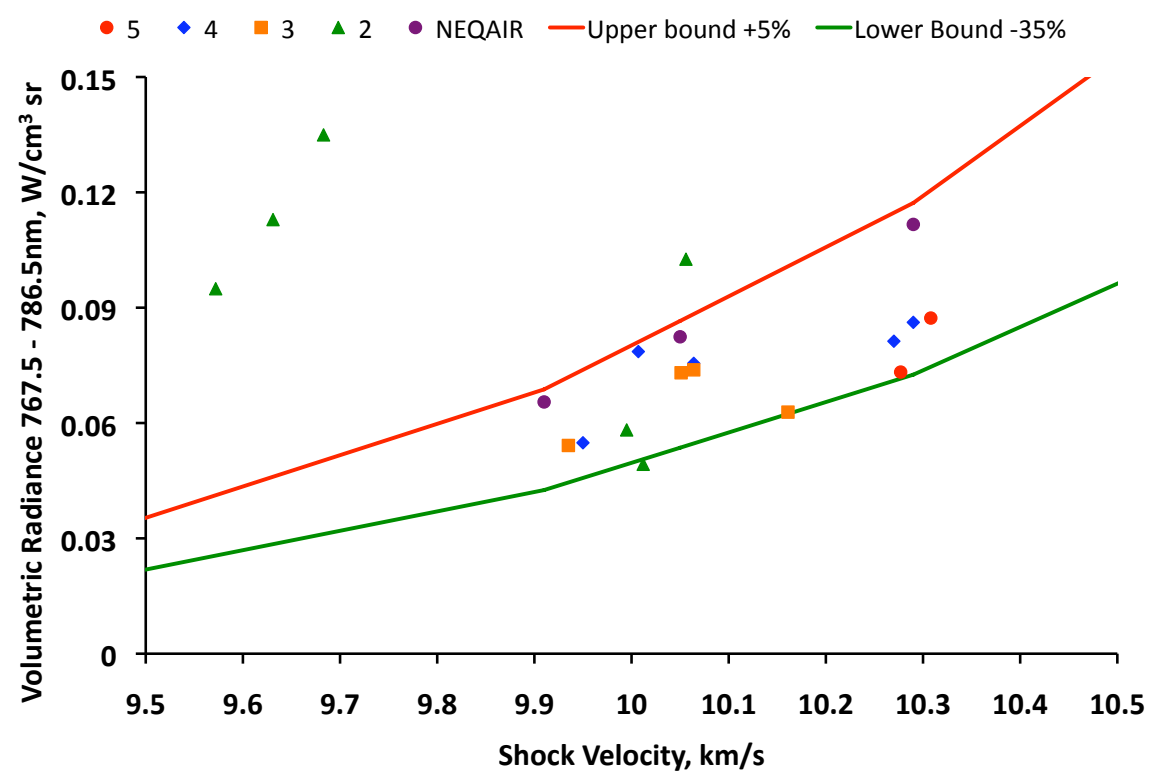

(a)

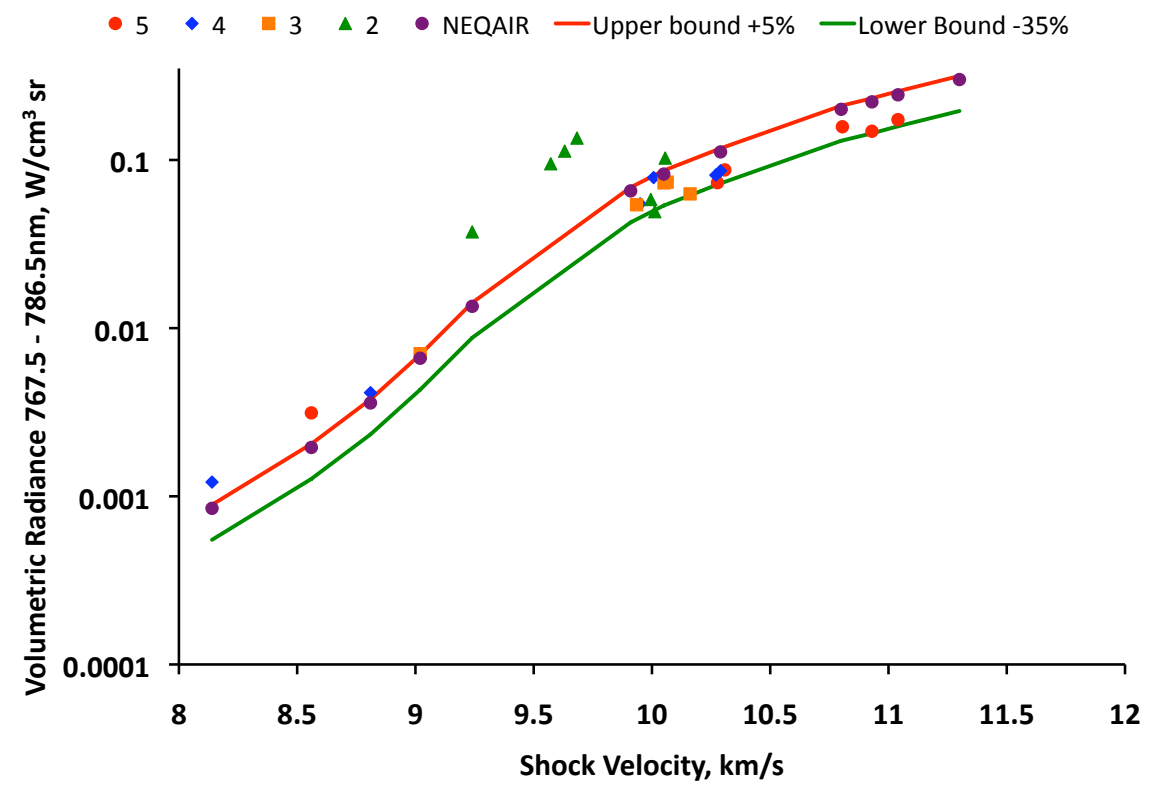

(b)

Figure 8. Vis/NIR comparisons of NEQAIR simulations and Test Campaign 47 and 50 EAST Experiments for the $777 \mathrm{~nm}$ oxygen line on a (a) linear scale, (b) and, log-scale. 


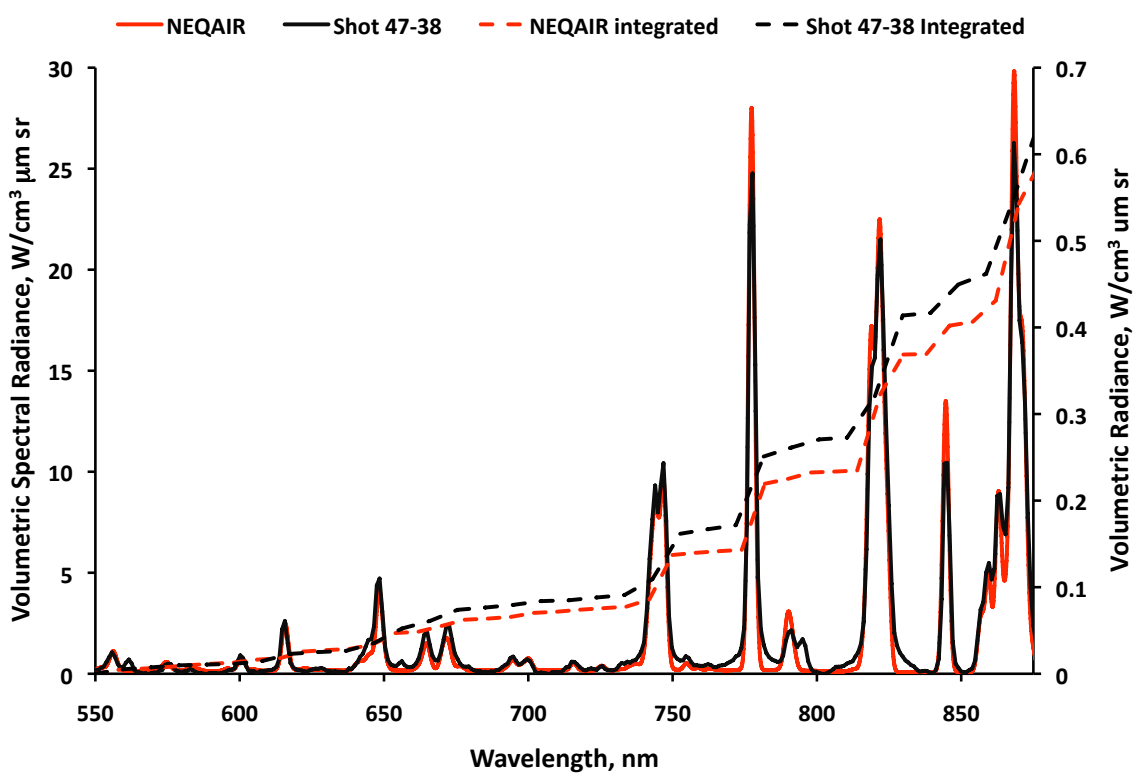

Figure 9. Comparison of NEQAIR and EAST for the $777 \mathrm{~nm}$ oxygen line with the background continuum subtracted.

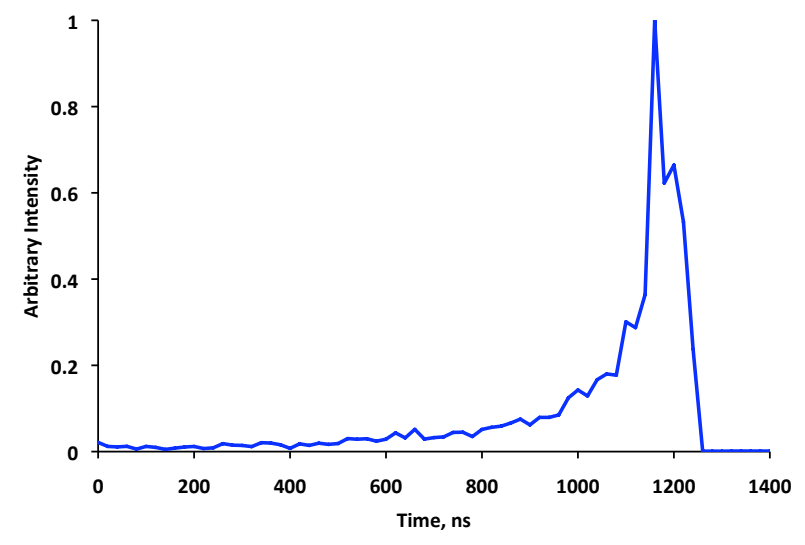

(a)

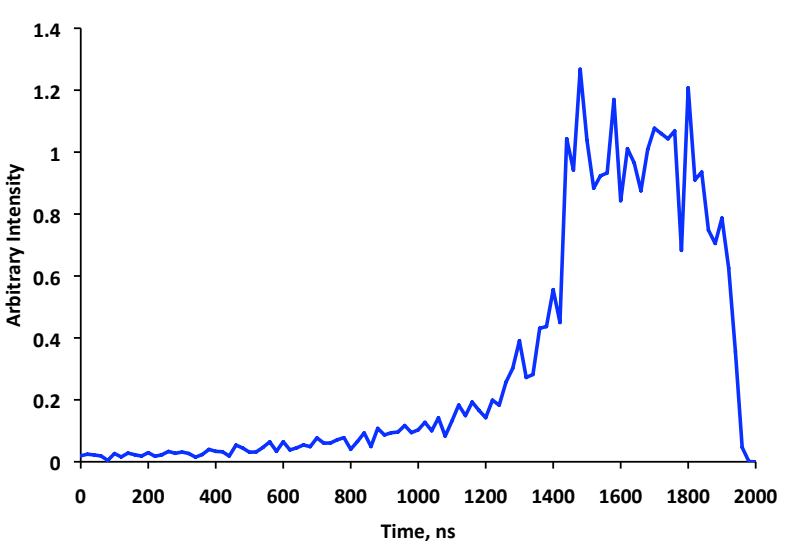

(b)

Figure 10. Examples of the gate opening for the Vis/NIR spectrometer at the settings of (a) $100 \mathrm{ns,} \mathrm{and,} \mathrm{(b)}$ 500 ns. 
wavelength range, and then summed over all the spectral regions investigated. This process is represented by equation 2, where $\mathrm{U}_{t o t}$ is the total uncertainty, $n$ is the number of spectral ranges to be investigated, $I_{n}$ is the intensity for the ${ }^{n t h}$ spectral region, $I_{t o t}$ is the total intensity defined in equation 3 and $U_{n}$ is the uncertainty for the ${ }^{\text {nth }}$ spectral region.

$$
\begin{gathered}
U_{t o t}=\sum_{1}^{n} \frac{I_{n}}{I_{t o t}} \times U_{n} \\
I_{t o t}=\sum_{1}^{n} I_{n}
\end{gathered}
$$

The results of the analysis is shown in Table 3 . Table 3 shows for each spectral range analyzed, the upper and lower bounds of uncertainty scaled by the relative contribution of radiance emitted by that range to the total radiance. These scaled upper and lower certainties are then summed and give the upper uncertainty bound to be $+8.9 \%$, and the lower uncertainty bound to be $-32.4 \%$. This indicates that NEQAIR generally provides more of an upper bound prediction of the EAST radiance emitted by atomic and molecular species. It should also be pointed out that these uncertainty bounds do not include any uncertainty related to the background continuum. Displaying the data in this way also provides a way to compare with other current or future simulations and experiments.

It is also of interest to look at the data in the slower and faster regions of the shot data, such as $8-9.5 \mathrm{~km} / \mathrm{s}$ and $10.5-12 \mathrm{~km} / \mathrm{s}$. The analysis in these regions is less robust due to fewer data points. Additionally, at lower speeds the uncertainty on the integrated value is greatly increased due to a decreasing signal-to-noise ratio. However, a preliminary investigation has taken place to ascertain the uncertainty for these off-nominal speed ranges. For the range of $8-9.5 \mathrm{~km} / \mathrm{s}$, the upper uncertainty bound was found to be approximately $+130 \%$, and the lower uncertainty bound to be approximately $+40 \%$. For the range of $10.5-12 \mathrm{~km} / \mathrm{s}$, the upper uncertainty bound was found to be approximately $-22 \%$, and the lower uncertainty bound to be approximately $-35 \%$. This indicates that the emitted radiance relationship with velocity seen on EAST is not completely captured by NEQAIR. This could be due to not including, or incorrectly modeling, some effects that are changing with velocity and/or flow temperature, such as the boundary layer.

Table 3. Upper and lower bounds for NEQAIR compared to EAST spectral intensities (background continuum subtracted).

\begin{tabular}{cccccc}
\hline \hline $\begin{array}{c}\text { Wavelength } \\
\text { Range, nm }\end{array}$ & $\begin{array}{c}\text { NEQAIR } \\
\text { Radiance @ 10km/s } \\
\mathbf{W} / \mathbf{c m}^{3} \mathbf{s r}\end{array}$ & $\begin{array}{c}\text { Upper } \\
\text { Uncertainty } \\
\text { Bound }\end{array}$ & $\begin{array}{c}\text { Scaled } \\
\text { Uncertainty } \\
\text { Upper }\end{array}$ & $\begin{array}{c}\text { Lower } \\
\text { Uncertainty } \\
\text { Bound }\end{array}$ & $\begin{array}{c}\text { Scaled } \\
\text { Uncertainty } \\
\text { Lower }\end{array}$ \\
\hline $146-153$ & 0.163 & $0 \%$ & $0.0 \%$ & $-40 \%$ & $-7.2 \%$ \\
$170.7-182.2$ & 0.15 & $-10 \%$ & $-1.7 \%$ & $-30 \%$ & $-5.0 \%$ \\
$310-470$ & 0.07 & $45 \%$ & $3.5 \%$ & $-25 \%$ & $-1.9 \%$ \\
$470-500$ & 0.0027 & $130 \%$ & $0.4 \%$ & $0 \%$ & $0.0 \%$ \\
$507.5-556$ & 0.007 & $125 \%$ & $1.0 \%$ & $0 \%$ & $0.0 \%$ \\
$567-648$ & 0.023 & $70 \%$ & $1.8 \%$ & $-3 \%$ & $-0.1 \%$ \\
$664-704$ & 0.013 & $68 \%$ & $1.0 \%$ & $0 \%$ & $0.0 \%$ \\
$735-755$ & 0.061 & $-10 \%$ & $-0.7 \%$ & $-36 \%$ & $-2.4 \%$ \\
$767.5-786.5$ & 0.08 & $5 \%$ & $0.4 \%$ & $-35 \%$ & $-3.1 \%$ \\
$804-834$ & 0.145 & $7 \%$ & $1.1 \%$ & $-36 \%$ & $-5.8 \%$ \\
$850-885$ & 0.19 & $10 \%$ & $2.1 \%$ & $-33 \%$ & $-6.9 \%$ \\
\hline Sum: & 0.9047 & \multicolumn{5}{|c|}{} & $\mathbf{- 3 2 . 4 \%}$ \\
\hline \hline
\end{tabular}

\section{E. Possible Explanation for Background Continuum Radiation}

The bound-free transitions are responsible for the background continuum radiation found in NEQAIR. However, the level of continuum is significantly lower than has been measured in experiments. One possible 


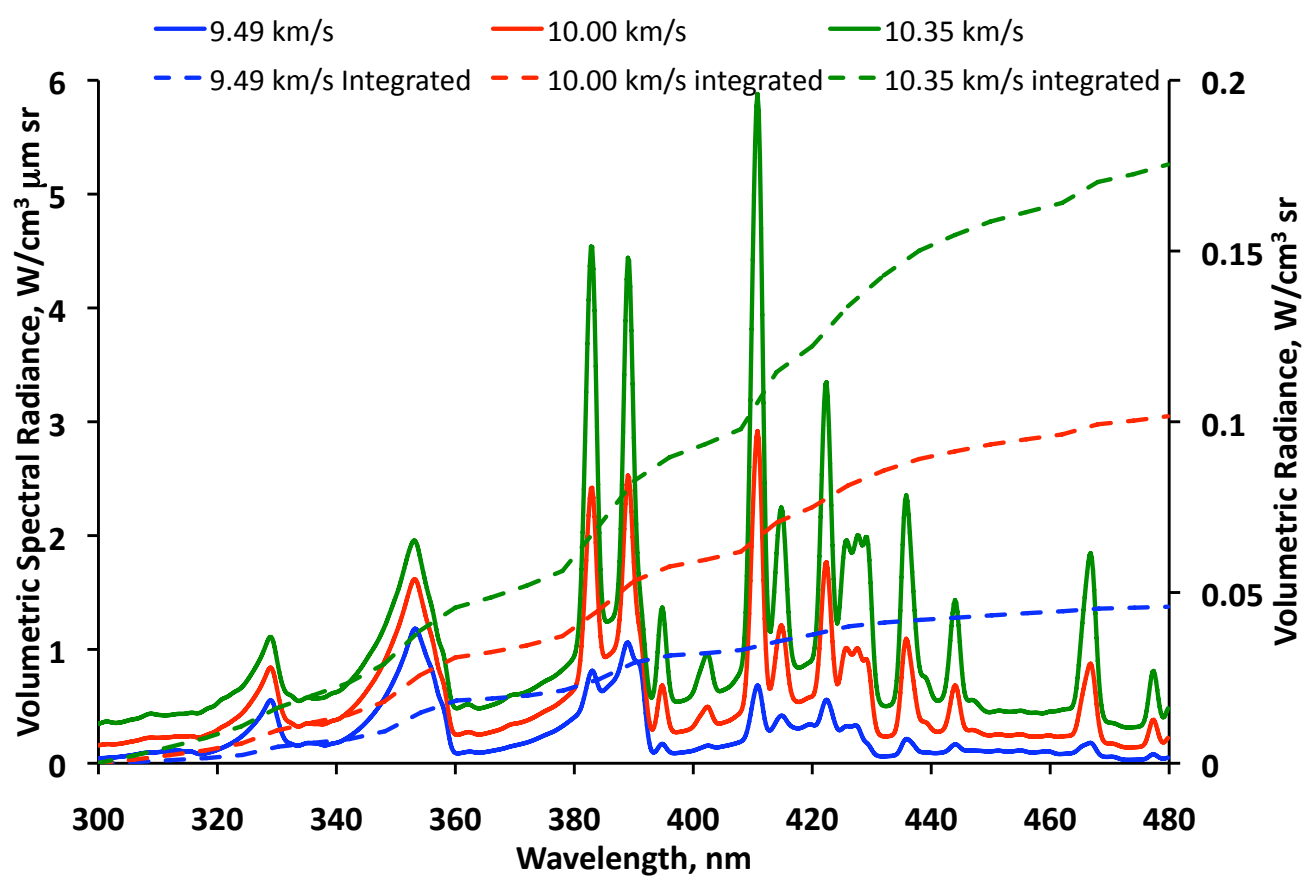

Figure 11. The significance of velocity variation on the change of radiance for $9.49 \mathrm{~km} / \mathrm{s}, 10.00 \mathrm{~km} / \mathrm{s}$ and $10.35 \mathrm{~km} / \mathrm{s}$.

explanation for this is due to the fact the shock front is decelerating, and that the test gas have been processed by different shock speeds. Furthermore, because the gas has been compressed behind the shock, the various parts of the gas have also relaxed for different times. This paper has shown that the agreement of the atomic and molecular radiation is generally very good when compared to the NEQAIR solution based on the $\mathrm{CEA}^{10}$ calculations using the shock front velocity.

In an attempt to better simulate the flow in the EAST facility, a 1-D NEQAIR shock deceleration analysis was undertaken. It was hoped that the results of such an analysis would provide some insights into the significant under-prediction of the background continuum on EAST. In the EAST experiment the shock is decelerating significantly. In the example analyzed in this paper, the shock decelerates from $10.75 \mathrm{~km} / \mathrm{s}$ to $10 \mathrm{~km} / \mathrm{s}$ over a distance of $150 \mathrm{~cm}$. Therefore, there is gas in the test section that has been shocked at speeds ranging from $10 \mathrm{~km} / \mathrm{s}$ up to approximately $10.5 \mathrm{~km} / \mathrm{s}$. This $5 \%$ change in shock speed is very significant in terms of predicting the EAST emitted radiance, as demonstrated in Fig. 11. The background continuum radiation is proportional to the electron number density (as it's a recombination process) which increases dramatically with shock speed, as shown in Fig. 12. The atomic species radiation is also proportional to shock speed (as translational temperature is also increasing), but to a lesser extent. However, the increase in atomic radiation is somewhat countered by the decreasing atomic nitrogen number density. The atomic nitrogen number density is decreasing due to ionization increasingly having a greater effect than the dissociation of molecular nitrogen. Therefore, the relative contribution of the background continuum radiation compared to the spectral radiation increases. The analysis presented here should be viewed as a first step and is not meant to be definitive. Further analysis is required and highlights the need for unsteady 2-D axisymmetric calculations, which would also include thermal losses to the wall, boundary layer effects and better capture the deceleration of the shock and unsteady flow processes.

The simulations are performed using the version of NEQAIR that uses gaunt factors for the calculation of the bound-free emissions. The background continuum is calculated by running NEQAIR with only the bound-free transitions turned on. It is the bound-free transitions that are responsible for the background continuum in NEQAIR. With this value known, the ratio compared to the total emitted radiation can be calculated. This calculation does have its limitations, such as not accounting for any thermal losses to the wall or absorption by the boundary layer.

The basic approach to the analysis is shown in Fig. 13. Several different shot locations were included in the analysis, as is indicated in Fig. 13(a). For each shock location, the velocity of the gas behind the shock 


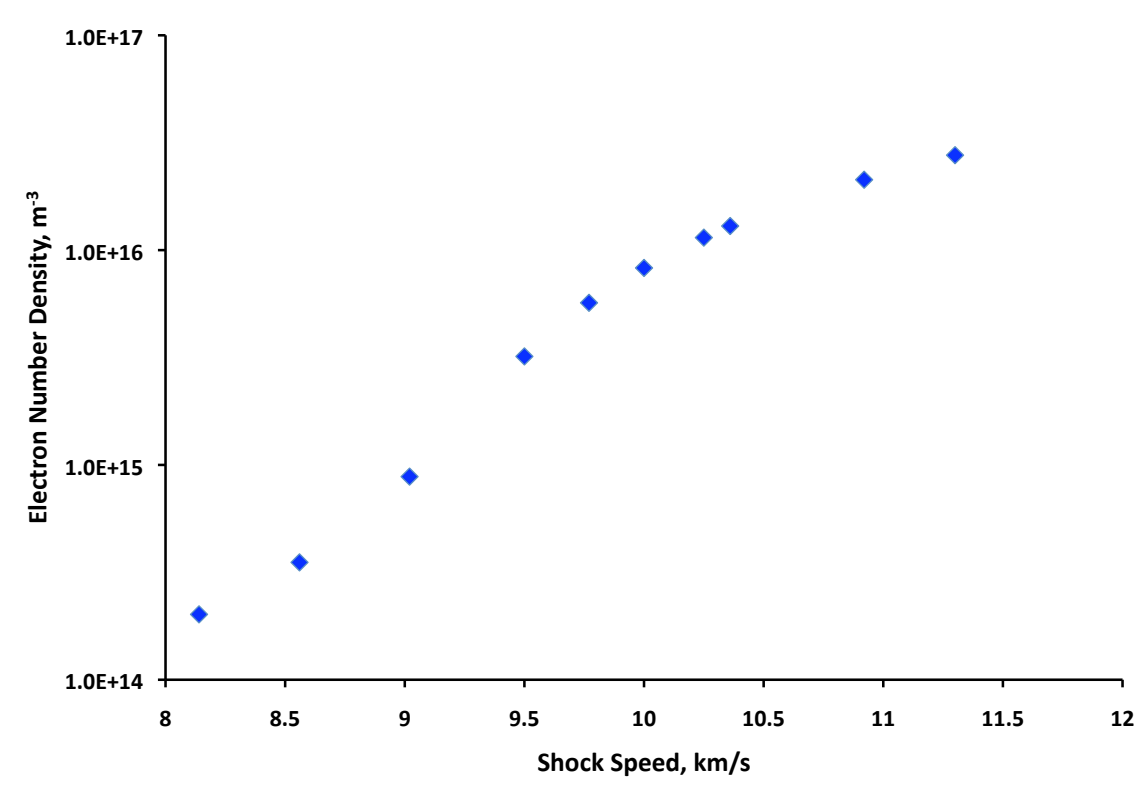

Figure 12. Equilibrium electron number density verses velocity as calculated by CEA.

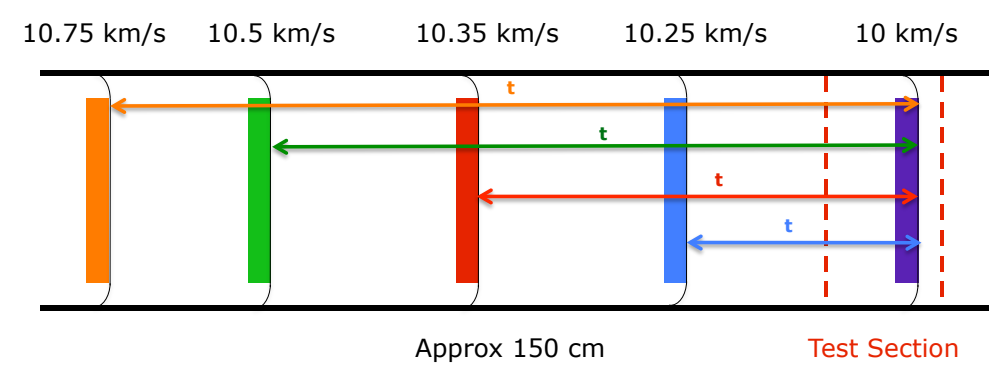

(a)

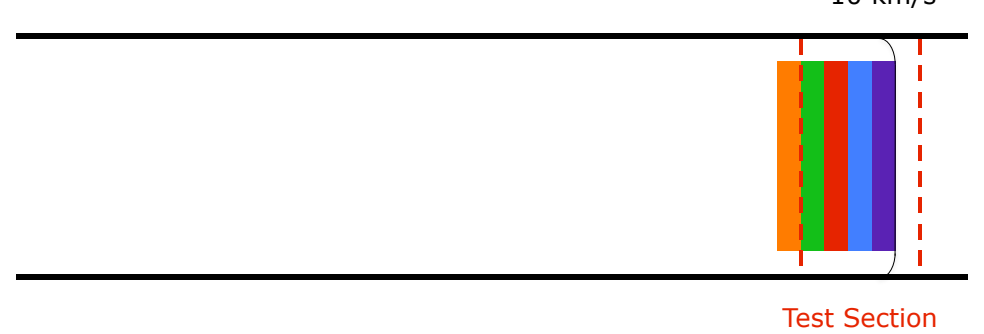

(b)

Figure 13. Schematic of the 1-D NEQAIR EAST analysis attempting to model the deceleration of the shock. 
is calculated by a 1-D shock calculation. The deceleration of the shock and the time it takes for the shock to travel from this particular point to the test section are known because they are quantities that can be measured from experiment. Therefore, with velocity, acceleration and time, the distance this shocked gas travels is calculated. It can then be determined whether this gas would arrive in the test section when the spectrometer is gated and the measurement taken, as indicated in Fig. 13(b). Furthermore, knowing the time it has taken to travel to the test section, the properties of this gas are calculated based on a relaxation process behind a shock. The mutation code developed by Magin et al. is used to calculate the relaxation process. ${ }^{12}$ The emitted radiance is calculated, and "smeared" over the distance that corresponds to its velocity and the gate width used in the experiment.

Fig. 14 shows the results from this analysis. The result from assuming a $10 \mathrm{~km} / \mathrm{s}$ shock and no deceleration is shown in red. The discrete plateaus correspond to the different locations where the shock was analyzed and the ratio is constant at approximately $34 \%$. The result from attempting to model the shock deceleration is shown in green. Here the evolution of the emitted radiation against distance can be seen. The percentage of continuum radiation compared to total radiation has increased to $46 \%$. An example of this ratio, shown in blue, obtained from EAST measurements shows that there is significant improvement in the agreement of the ratio. However, there is a significant level of noise due to the integration and background continuum subtraction over a relatively small number of pixels. The two points around $785 \mathrm{~cm}$ that are significantly higher than the shock deceleration simulation may indicate that the flow has not reached an adequate level of equilibrium at this location. Furthermore, it would be beneficial to extend the calculations to individually analyze several shots in this manner.

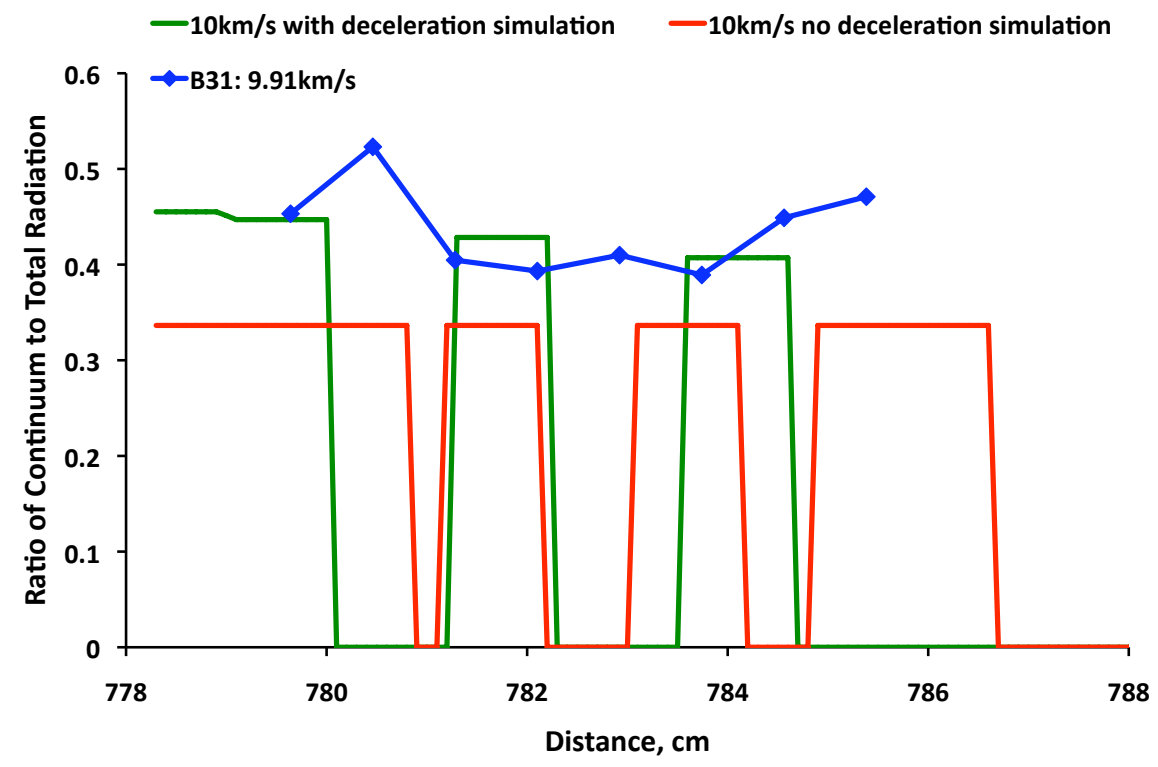

Figure 14. Preliminary qualitative results for the 1-D NEQAIR EAST analysis attempting to model the deceleration of the shock integrated over $310-470 \mathrm{~nm}$.

\section{Comparison of EAST and X2}

The facility and methodology behind both X2 and EAST testing is similar, and thus they are a good source of data comparison, especially when it is considered that there are several common testing conditions. However, it is important to note there are two main differences between how the facilities operate and measure radiation. In the EAST, the radiation is analyzed through a window in the side of the shock tube, whereas in X2, the radiation is measured behind the shock after it exits the tunnel. This effectively means that in EAST, the radiation passes through the boundary layer, whereas in X2, the radiation passes through an expansion fan structure (of which some of the flow is from the tube boundary layer) caused by the change in area as the flow propagates into the dump tank. There is the intention to conduct experiments on the $\mathrm{X} 2$ facility looking through the boundary layer to provide a more direct comparison with the EAST results. Due to the boundary layer being less significant on the X2 facility where the measurement is taken, the 


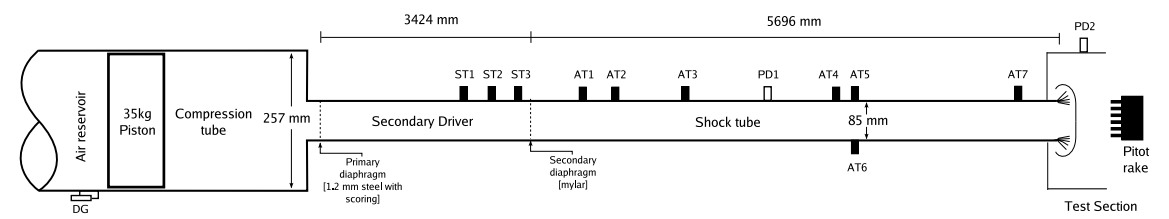

Figure 15. Schematic of X2 facility.

effect of tube contamination from the walls of the facility may be mitigated. This could provide useful information with respect to the EAST facility, as it is believed that the wall provides some contaminating species to the flow. The level of accuracy in which the shock speed can be measured is also different for the two facilities. In EAST the shock speed is quoted to within 0.3-0.5\%, whereas on X2, the shock speed is quoted to within $2.5 \%$. However, the $\mathrm{X} 2$ databox did not trigger for several of the shots for the 26.6 Pa (0.2 Torr) condition. Therefore, in these instances the uncertainty in the shock speed is much higher, around $5 \%$ and estimated based on the shock location on the ICCD. The shock speed for these cases also generally came out higher. This may be due to the opening time of the spectrometer gate, and accurately identifying the end of the tunnel and shock front location. The best estimate of their shock speeds is to be within the scatter of the shots where the databox did trigger. Furthermore, the process for the calculation of the X2 shock speeds presented in this paper is different from previous analyses. Previously the averaged velocity between pressure sensors at 5 and at7 (refer to Fig. 15 and Table 4 for transducer locations) was quoted as the shock speed. The methodology used in the present analysis for X2 is similar to the approach used in EAST. The time of arrival for pressure transducers at4, at5 and at7 are determined and then fitted with a 2nd-order polynomial. The derivative of the polynomial function is then extrapolated to the shock front location, and the velocity calculated. This approach gives a better representation of the shock velocity at the time of the test, and thus providing a better source of comparison with EAST. However, the uncertainty in the measurement is expected to be greater in $\mathrm{X} 2$ as a result of having to extrapolate the function as well as having fewer transducers in the polynomial fit ( 3 on X2 vs 11 in EAST).

Table 4. Location of wall pressure sensors in X2 (dimensions in $\mathrm{mm}$ ).

\begin{tabular}{cccc}
\hline \hline Secondary Driver & ST1 -2572 & ST2 - 2804 & ST3 - 3036 \\
\hline Shock Tube & AT1 -3955 & AT2 - 4205 & AT3 -6019 \\
& AT4 -7865 & AT5 -8045 & AT6 - 8660 \\
\hline \hline
\end{tabular}

\section{A. Analysis and Comparison}

One issue for the presentation of the experimental data for comparison purposes is the units to be used for the radiative intensity. When the intensity is calibrated, the units are given as an absolute spectral radiance, $\mathrm{W} / \mathrm{cm}^{2} . \mu \mathrm{m} . \mathrm{sr}$. This represents the radiance emitted from an imaged slice of the test gas. However, in terms of comparing the EAST and X2 results, these units may not be directly compared due to the tunnels having different diameters. Furthermore, the core-flow on the X2 facility is reduced even further due to the expansion fan process. To convert this radiance to a facility independent and comparable quantity, the spectral radiance needs to be corrected for the line of sight. Therefore, the radiative intensities may be expressed in terms of volumetric units, i.e. $\mathrm{W} / \mathrm{cm}^{3} . \mu \mathrm{m} . \mathrm{sr}$, , under optically thin assumptions. To make this conversion, the width of the radiating gas in which the measurement was integrated over needs to be known. As on the EAST facility the measurement is taken while inside the shock tube, this width can be said to be the width of the tube. This means that the boundary layer has been assumed to have a negligible thickness. For the benefit of comparison, a core width of X2 has been assumed. In a previous CFD analysis it was estimated that the minimum core width should be approximately $72.5 \mathrm{~mm}$. So for this analysis, this is assumed to be the lower limit. The upper limit is the width of the tunnel, which is $85 \mathrm{~mm}$. Therefore, for this preliminary analysis, the core width has been assumed to be simply the average of the upper and lower limit, $78.8 \mathrm{~mm}$. This value therefore has an uncertainty bound of $\pm 8 \%$, and should be considered as a very basic estimate of core flow until further CFD results have been obtained. It should also be noted that the 


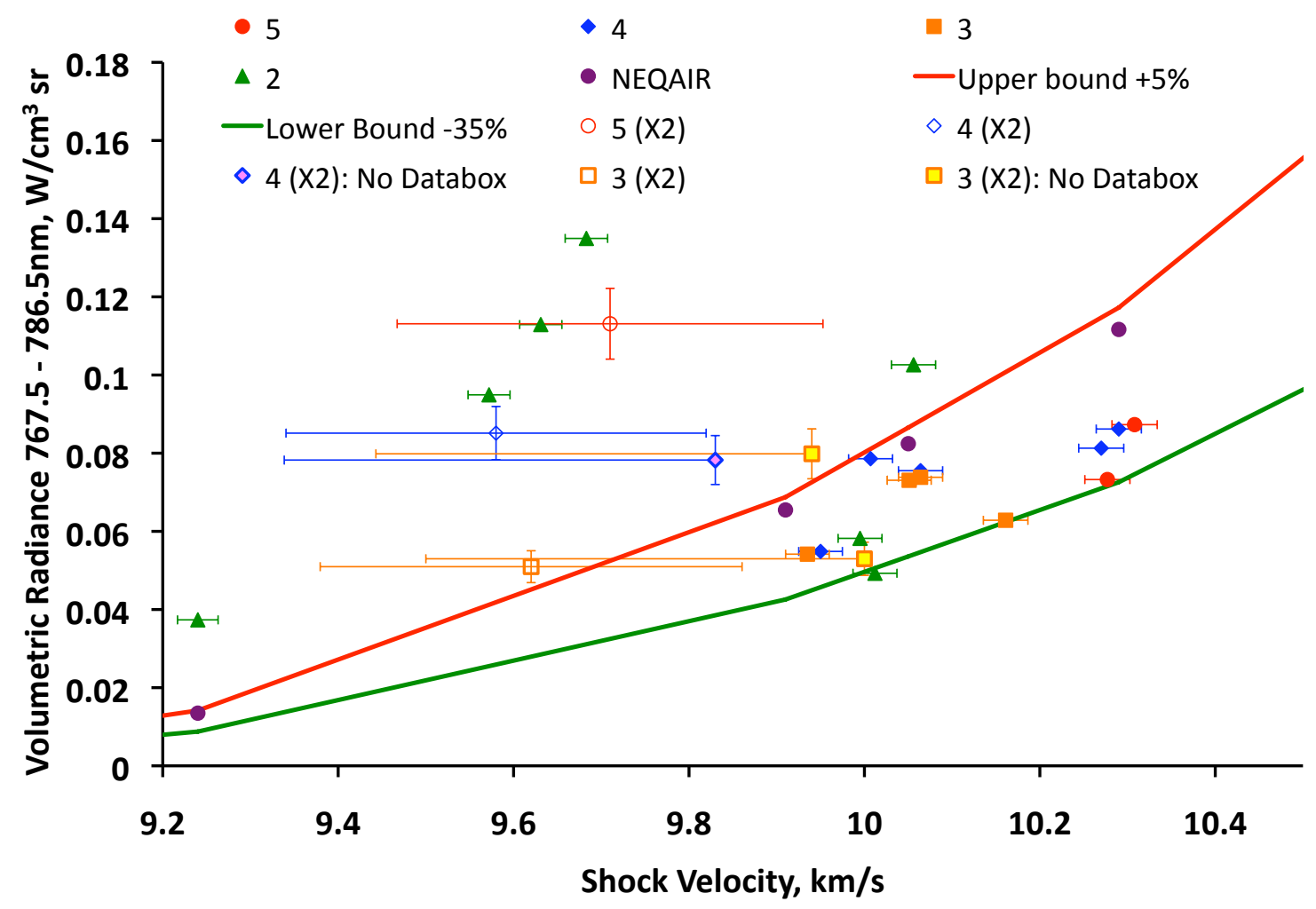

Figure 16. Preliminary comparison of EAST, X2 and NEQAIR results for the $777 \mathrm{~nm}$ oxygen line.

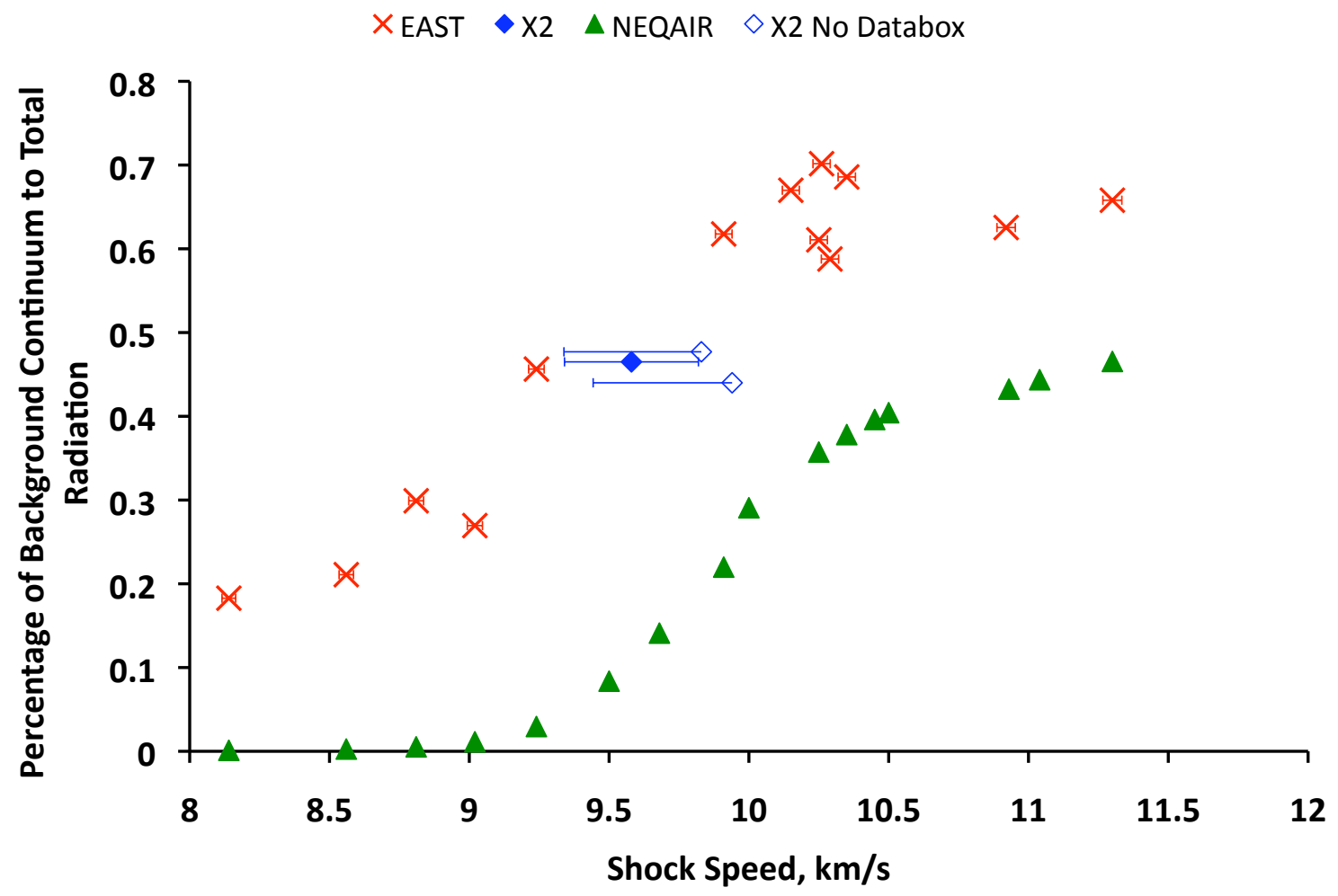

Figure 17. Comparison of percentage of background continuum to total radiation in UV/Vis from $340-430 \mathrm{~nm}$ for X2, EAST and NEQAIR. 
wavelength calibration of the X2 data had to be shifted to align with known spectral peaks (up to $20 \mathrm{~nm}$ depending on the spectral location). This transformation was required due to an error in the wavelength calibration of the grating. This would effect absolute calibration in regions where the calibration lamp or spectrometer response functions are not flat.

Due to the small number of shots on X2, and an increased uncertainty with regards to the absolute radiance and shock speed, there is not a sufficient number of shots to do a thorough statistical analysis of the data. Therefore, there is more benefit in conducting qualitative comparisons. An example of such a comparison would be to compare the relative contribution of the background continuum radiation to the total emitted radiation, as shown in Fig. 17. This confirms that the larger than expected background continuum is not an artifact unique to the EAST facility. Furthermore, it can be seen that the relative contribution of the background continuum is very similar in magnitude between X2 and EAST. Especially if the bias in the $\mathrm{X} 2$ data is considered that indicates that the quoted velocity is an upper-bound. Although, it should be pointed out that even though these results confirm that the background continuum has been measured on two different facilities, the ICCD used in both instances is the same. Therefore, it is plausible, although speculative, that the background continuum could potentially be an artifact of the camera. Another conclusion that can be drawn, is that the background continuum is unlikely to be due to the boundary layer. This can be concluded as the flow is not measured looking through the boundary layer on X2.

\section{Conclusion}

This paper has presented results from the EAST facility for conditions ranging from $8-11.5 \mathrm{~km} / \mathrm{s}$ and 26.6 Pa. The higher speed conditions were enabled through use of a buffer gas arrangement on EAST. A comprehensive comparison with NEQAIR has been conducted, and various spectral features and trends analyzed. The results from the EAST facility show very good agreement in the VUV, UV/Vis and Vis/NIR regions of the spectrum. The Vis/NIR spectrometer gate opening has been measured and shown to be the cause of the non-reciprocity presented previously. ${ }^{4}$ Furthermore, the slow opening of the gate is also responsible for some level of spatial smearing. Due to issues with the IR camera, these results have not yet been analyzed. A criteria for determining the quality of the equilibrium plateau region has been developed. This criteria has then been used with the NEQAIR simulations to provide upper and lower bounds for NEQAIR for three different speed regimes. The most thorough analysis has been conducted for the most flight relevant speed regime of $9.5 \mathrm{~km} / \mathrm{s}$ to $10.5 \mathrm{~km} / \mathrm{s}$, with the upper and lower bounds being $+8.9 \%$ and $-32.4 \%$ respectively. A less robust (due to fewer data points) and preliminary analysis was conducted for the $8-9.5 \mathrm{~km} / \mathrm{s}$ and $10.5-12 \mathrm{~km} / \mathrm{s}$ speed regimes. The upper and lower bounds were $+130 \%$ and $+40 \%$ for the slower speeds, and $-22 \%$ and $-35 \%$ for the faster speeds. There appears to be a discrepancy between the experimentally measured and simulated radiation/shock speed relationship, with the error bias shifting from positive (overpredicted) to negative (underpredicted) as velocity increases. A preliminary comparison with the X2 facility results was also presented. However, due to the small number of shots on X2, and an increased uncertainty with regards to the absolute radiance and shock speed, there is not a sufficient number of shots to do a thorough statistical analysis of the data. Therefore, more qualitative comparisons are presented. The background continuum has been analyzed on a relative basis in both the EAST and X2 facilities and found to be quite similar. The results from a 1-D NEQAIR shock deceleration analysis suggest that not including the effect of the shock decelerating, and thus sections of the gas being shocked at higher speeds, could be the cause of the discrepancy between the simulated and experimentally measured level of continuum. 


\section{Future Work}

The engineering-level NEQAIR calculations presented in this paper are only a first attempt at modeling the shock deceleration phenomenon However, despite the limitations of the simulation, the results have shown that modeling the deceleration of the shock could explain some of the current discrepancies related to the continuum. Therefore, future efforts aimed at constructing and implementing a 2-D/axi-symmetric simulation of the EAST flow should prove to be highly beneficial. The present analysis has also shown the importance of analyzing both CFD and experimental results together. In particular, the benefits obtained by expanding the experimental scope to include off-nominal design conditions to provide a comprehensive dataset to better validate the simulations. There is scope for the optimization of EAST buffer design to provide even higher speeds. Furthermore, the focus of the buffer testing thus far has been directed towards Earth entries. Future testing could also provide high speed entry data for other atmospheres, such as Mars, Venus and Titan. For future testing in the IR region of the spectrometer, modifications to the optical arrangement could reduce the amount of light entering the spectrometer and help provide more robust IR data.

\section{Acknowledgments}

The author would like to thank the following people: Nagi Mansour, Parvis Moin, Gianluca Iaccarino for all their efforts and support for my Postdoctoral Research Fellow position at Stanford University and NASA Ames Research Center. Ramon Martinez and Hai Le for their efforts with obtaining the experimental data from NASA Ames Research Center's EAST facility. Brett Cruden and Dinesh Prabhu were supported by Contract NNA04BC25C to ELORET Corporation from the Entry Systems and Technology Division, NASA Ames Research Center. Support and encouragement of the work by Joseph Olejniczak (Proj. Mgr., CEV $\mathrm{CAP}$ ) is acknowledged.

\section{References}

${ }^{1}$ Johnston, C., "A Comparison of the East Shock-Tube Data with a New Air Radiation Model," AIAA, Reno, Nevada, January 2008, pp. AIAA 2008-1245.

${ }^{2}$ Bose, D., McCorkle, E., Thompson, C., Bogdanoff, D., Prabhu, D., Allen, G., and Grinstead, J., "Analysis and Model Validation of Shock Layer Radiation in Air," AIAA, Reno, Nevada, January 2008, pp. AIAA 2008-1246.

${ }^{3}$ Panesi, M., Magin, T., Bourdon, A., Bultel, A., and Chazot, O., "Analysis of the Fire II Flight Experiment by means of a Collisional Radiative Model," Journal of Thermophysics and Heat Transfer, 2009, Accepted for publication.

${ }^{4}$ Cruden, B., Martinez, R., Grinstead, J., and Olejniczak, J., "Simultaneous Vacuum-Ultraviolet Through Near-IR Absolute Radiation Measurement with Spatiotemporal Resolution in An Electric Arc Shock Tube," 41st AIAA Thermophysics Conference, Vol. AIAA-2009-4240, San Antonio, Texas, 2009.

${ }^{5}$ Whiting, E., Yen, L., Arnold, J., and Paterson, J., "NEQAIR96, Nonequilibrium and Equilibrium Radiative Transport and Spectra Program: Users Manual,," Technical Report NASA RP-1389, Ames Research Center, Moffet Field, Moffet Field, 1996.

${ }^{6}$ Johnston, C., Nonequilibrium Shock-Layer Radiative Heating for Earth and Titan Entry, Ph.D. thesis, Virginia Polytechnic Institute and State University, Virginia, 2006.

${ }^{7}$ Grinstead, J. H., Olejniczak, J., Wilder, M. C., Bogdanoff, D. W., Allen, G. A., and Lilliar, R., "Shock-heated Air Radiation Measurements at Lunar Return Conditions," 46th AIAA Aerospace Sciences Meeting and Exhibit, 7-10 January 2008, Reno, Nevada 2008, pp. AIAA 2008-1244.

${ }^{8}$ Bogdanoff, D. W., "Shock tube experiments for Earth and Mars entry conditions," Non-equilibrium gas dynamics, from physical models to hypersonic flights, Vol. Von Karmen Institute Lecture Series, 2008.

${ }^{9}$ Bose, D., McCorkle, E., Bogdanoff, D. W., and Allen Jr., G., "Comparisons of air radiation model with shock tube measurements. AIAA Paper 2009-1030," 47th AIAA Aerospace Sciences Meeting, Orlando, Florida, 2009.

${ }^{10} \mathrm{McBride}, \mathrm{B}$. and Gordon, S., "Computer Program for Calculation of Complex Chemical Equilibrium Compositions and Applications I. Analysis," NASA RP-1311, NASA Glenn, October 1994.

${ }^{11}$ Ralchenko, Y., Kramida, A., Reader, J., and NIST ASD Team, "NIST Atomic Spectra Database (version 3.1.5),," [online]. available: http://physics.nist.gov/asd3 National Institute of Standards and Technology, NASA Glenn, Gaithersburg, MD, 2010.

${ }^{12}$ Magin, T., Caillault, L., Bourdon, A., and Laux, C., "Nonequilibrium radiation modelling for Huygens entry," Journal of Geophysical Research, Vol. 111, 2006. 